\title{
AN EXAMPLE OF AN INFINITE STEINER TREE CONNECTING AN UNCOUNTABLE SET
}

\author{
EMANUELE PAOLINI, EUGENE STEPANOV, AND YANA TEPLITSKAYA
}

\begin{abstract}
We construct an example of a Steiner tree with an infinite number of branching points connecting an uncountable set of points. Such a tree is proven to be the unique solution to a Steiner problem for the given set of points. As a byproduct we get the whole family of explicitly defined finite Steiner trees, which are unique connected solutions of the Steiner problem for some given finite sets of points, and with growing complexity (i.e. the number of branching points).
\end{abstract}

\section{INTRODUCTION}

In this paper we construct an explicit and rather natural example of an infinite tree connecting some "fractal" set of points (in fact, homeomorphic to a Cantor set, and in particular compact, uncountable and totally disconnected) in the optimal way in the sense that it solves the Steiner problem for this set of points. The Steiner problem which has various different but more or less equivalent formulations, is that of finding a set $S$ with minimal length (one dimensional Hausdorff measure $\mathcal{H}^{1}$ ) such that $S \cup A$ is connected, where $A$ is a given compact subset of a given complete metric space $X$. Namely, defined

$$
\mathcal{S} t(A):=\{S \subset X: S \cup A \text { is connected }\},
$$

one has to find an element of $\mathcal{S} t(A)$ with minimal length $\mathcal{H}^{1}$. This problem appeared in the work of V. Jarník and O. Kössler of 1934, but actually became famous later, after having been cited in the book of R. Courant and H. Robbins "What is Mathematics?" where it has been linked to the name of J. Steiner. Usually it is stated in the case when the ambient space $X$ is the Euclidean space $\mathbb{R}^{n}$ (or even the Euclidean plane $\mathbb{R}^{2}$ ), the set $A$ (interpreted, say, as the set of cities to be connected by roads) is finite, while the solutions (interpreted in this case as the sets of roads connecting the given cities in the optimal way) are required a priori to be the finite sets of line segments (for the case when $A$ is the set of three points in the plane, this is nothing but the famous problem posed by P. Fermat already in the seventeenth century). Even in such a formally restricted setting this problem is subject of active study until nowadays, and presents still a lot of open problems (for the extremely extensive literature on the subject see, for instance $[2,1]$ and references therein). The general setting of this problem as stated above (i.e. with $X$ generic metric space, $A$ not necessarily finite, and without any a priori restriction on the class of minimizers) has been recently studied in [5], in which under rather mild assumptions on the ambient space $X$ (which anyhow are true in the Euclidean space setting) it is shown that every solution $S$ having finite length $\mathcal{H}^{1}(S)<+\infty$ has the following properties:

- $S \cup A$ is compact;

The work of the second and third authors has been sponsored by the St.Petersburg State University grants \#6.38.670.2013 and \#6.38.223.2014 and by the Russian government grant NSh1771.2014.1. The work of the second author was also partially financed by GNAMPA, by RFBR grant \#14-01-00534 and by the project 2010A2TFX2 "Calcolo delle variazioni" of the Italian Ministry of Research. 
- $S \backslash A$ has at most countably many connected components, and each of the latter has strictly positive length;

- $\bar{S}$ contains no loops (homeomorphic images of $S^{1}$ );

- the closure of every connected component of $S$ is a topological tree (a connected, locally connected compact set without loops) with endpoints on $A$ (so that in particular it has at most countable number of branching points), and with at most one endpoint on each connected component of $A$ and all the branching points having finite order (i.e. finite number of branches leaving them);

- if $A$ has a finite number of connected components, then $S \backslash A$ has finitely many connected components, the closure of each of which is a finite geodesic embedded graph with endpoints on $A$, and with at most one endpoint on each connected component of $A$;

- for every open set $U \subset X$ such that $A \subset U$ one has that the set $\bar{S}:=S \backslash U$ is a subset of a finite geodesic embedded graph. Moreover, for a.e. $\varepsilon>0$ one has that for $U=\{x: \operatorname{dist}(x, A)<\varepsilon\}$ the set $\bar{S}$ is a finite geodesic embedded graph (in particular, it has a finite number of connected components and a finite number of branching points).

Thus, if $S$ is a solution to the Steiner problem for the given set $A$, then $\Sigma:=S \cup A$ also does not containing loops, unless of course $A$ itself contains loops. In this case $\Sigma$ is usually called Steiner tree, and, further, it is called indecomposable, when $\Sigma \backslash A$ is connected. It is worth mentioning that rather few explicit examples of solutions to the Steiner problem are known, and the known examples are mainly limited to the case when $A$ is a finite set. In fact, while some necessary conditions for a given set to be optimal are quite easy to obtain, it is usually quite hard to prove that the given set is optimal, and even harder to prove the uniqueness of the Steiner set (in fact, in general the solutions may be non unique, as can be easily seen on the example when $A$ is the set of vertices of a square). A promising method to deal with such problems has been proposed in [4]: although this method is not universal, sometimes it allows to prove the optimality of the concrete set. In this paper we provide an explicit example of a unique solution $S_{\infty}$ to a Steiner problem for some given set of points $A_{\infty} \subset \mathbb{R}^{2}$ of "fractal" type. The set $S_{\infty}$ is connected and disjoint from $A_{\infty}$, and thus $\Sigma_{\infty}:=S_{\infty} \sqcup A_{\infty}$ is an indecomposable Steiner tree with infinitely many branching points (joined by countably many line segments meeting with equal angles of $2 \pi / 3)$. As a byproduct we get the whole family of explicitly defined unique connected solutions of the Steiner problem for some given finite sets of points which are finite binary trees (it is customarily to say that these Steiner trees have full topology [2]), and with growing complexity (i.e. the number of branching points). Note also that the existence of a (finite) Steiner tree in the plane with arbitrary (but finitely) many branching points follows from the abstract result from $[2]$.

The set $A_{\infty}$ consists of the root and uncountably many leafs of the tree. No segment of $S_{\infty}$ touches the leafs, while every leaf is an accumulation point of segments of $S_{\infty}$. The infinite tree $\Sigma_{\infty}$ is composed by a trunk of some length $\ell$ which splits into two branches of length $\lambda_{1} \ell$ both of which split further into two branches of length $\lambda_{1} \lambda_{2} \ell$ and so on. Our proof requires that the sequence $\left\{\lambda_{j}\right\}$ vanish rather quickly (in fact, al least be summable). It is an open question if in the case of a constant sequence $\lambda_{j}=\lambda$ (with $\lambda>0$ small enough) the same construction still provides a Steiner tree. This seems to be quite interesting since the resulting tree would be, in that case, a self-similar fractal. 


\section{Notation}

For a subset $D \subset E$ of a metric space $E$ we denote by $\bar{D}$ and $\partial D$ its closure and its topological boundary respectively, and by $\mathcal{H}^{k}(D)$ its $k$-dimensional Hausdorff measure, we set dist $(x, D):=\inf \{d(x, y): y \in D\}$ whenever $x \in E$, and denote by $(D)_{\varepsilon}:=\{x \in E$ : dist $(x, D)<\varepsilon\}$ its $\varepsilon$-neighborhood. By $B_{r}(x)$ we denote the open ball of radius $r$ with center $x \in E$. The Euclidean norm in $\mathbb{R}^{n}$ is denoted by $|\cdot|$.

For points $A, B$ in the plane we let $(A B),[A B]$ and $[A B)$ (or $(B A])$ stands for the respective line, line segment and the ray with endpoint $A$, while $|A B|:=|A-B|$. By $\angle(a, b)$ we denote the angle between the two rays $a$ and $b$ (or between the ray and the line, depending on the context). By $\triangle A B C$ we denote the triangle with vertices $A, B$ and $C$, and by $\angle A B C$ the angle at the vertex $B$. The notation for the angles and for their measure is the same.

\section{Construction}

For the sake of brevity we introduce the notation

$$
\mathcal{M}(A):=\left\{S \in \mathcal{S} t(A): \mathcal{H}^{1}(S) \leq \mathcal{H}^{1}\left(S^{\prime}\right) \text { for every } S^{\prime} \in \mathcal{S} t(A)\right\},
$$

for the set of solutions of the Steiner problem. Set also $\mathcal{S}(A):=\mathcal{H}^{1}(S)$, where $S \in \mathcal{M}(A)$.

We call Fermat point of the triangle $\triangle A B C$ the point minimizing the sum of distances from the three vertices of the triangle. Such a point is well-known to be unique and will be denoted by $F(A, B, C)$. When all the angles of the triangle do not exceed $2 \pi / 3$, the Fermat point is inside the triangle and all sides of the triangle are visible from Fermat point at the angle of $2 \pi / 3$.

Let $L>0$ be a given length and $\left\{\lambda_{j}\right\}$ be a given sequence of positive numbers. We construct three sequences of points: $\left\{x_{n}, y_{n}, z_{n}\right\}_{n=1}^{\infty} \subset \mathbb{R}^{2}$ by the following recursive procedure (see Figure 1):

- $y_{0}:=\left(-L+2 \lambda_{1} L, 0\right) \in \mathbb{R}^{2}$;

- $y_{1}:=(0,0) \in \mathbb{R}^{2}, x_{1}=\left(2 \lambda_{g(1)} L, 0\right) \in \mathbb{R}^{2}$ with $g(j):=\left\lfloor\log _{2} j+1\right\rfloor$;

- $z_{n}:=\left(x_{n}+y_{n}\right) / 2$ for $n \geq 1$;

- the points $x_{n}, x_{2 n}, x_{2 n+1}$ are the three vertices, listed in counter-clockwise order, of the equilateral triangle inscribed in the circle with center $z_{n}$ and radius $\left|x_{n}-z_{n}\right|$;

- $y_{n}:=2 \lambda_{g(n)} y_{\lfloor n / 2\rfloor}+\left(1-2 \lambda_{g(n)}\right) x_{n}$ for $n \geq 1$ (observe that in this way $\left.y_{n}=F\left(y_{\lfloor n / 2\rfloor}, x_{2 n}, x_{2 n+1}\right)\right)$.

Let $i:=g(j)$. The point $x_{j}$ will be called leaf of the $i$-th generation and the point $y_{j}$ is called Fermat point of the $i$-th generation. 


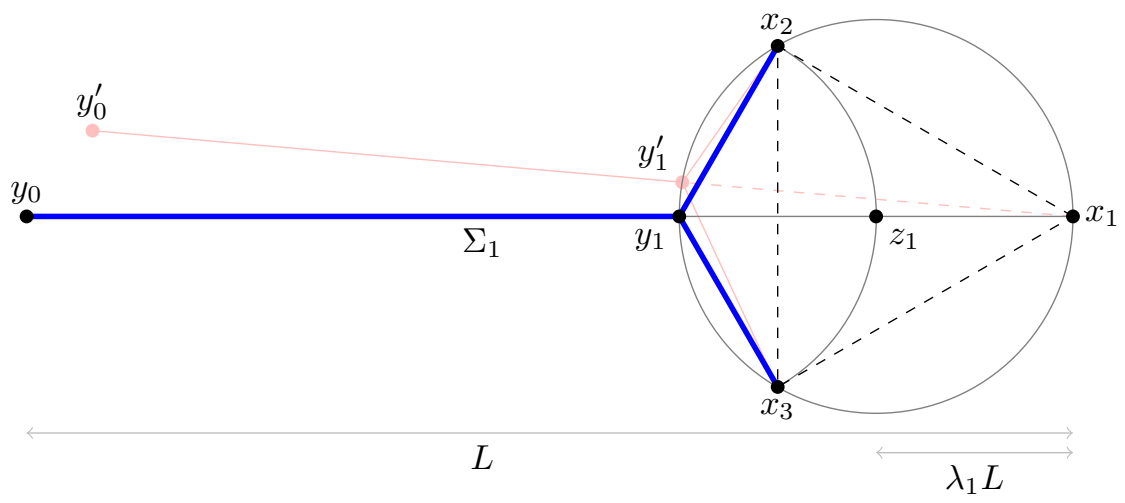

FiguRE 1. The first tripod in the construction of $\Sigma_{n}$

Let us define the following sets for $k=0,1, \ldots$ :

$$
\begin{aligned}
\sigma_{k} & :=\left[y_{0}, y_{1}\right] \cup \bigcup_{n=1}^{2^{k}-1}\left[y_{n}, y_{2 n}\right] \cup\left[y_{n}, y_{2 n+1}\right], \\
\Sigma_{k} & :=\sigma_{k} \cup \bigcup_{n=2^{k}}^{2^{k+1}}\left[y_{n}, x_{n}\right], \\
A_{k} & :=\left\{x_{2^{k}}, x_{2^{k}+1}, \ldots, x_{2^{k+1}-1}\right\} \text { the vertices of } \Sigma_{k}, \\
S_{\infty} & :=\bigcup_{k=1}^{\infty} \sigma_{k}, \quad \Sigma_{\infty}:=\bar{S}_{\infty}, \quad A_{\infty}:=\Sigma_{\infty} \backslash S_{\infty}, \\
\operatorname{Gen}\left(x_{p}\right) & :=\left\{x_{2 p}, x_{2 p+1}, x_{4 p}, \ldots, x_{2^{k} p}, \ldots, x_{2^{k} p+2^{k}-1} \ldots\right\} \\
& \text { the set of the descendants of vertex } x_{p}, \\
\operatorname{Gen}_{k}\left(x_{p}\right) & :=A_{k} \cap \overline{\operatorname{Gen}\left(x_{p}\right)} .
\end{aligned}
$$

Let us call $\Sigma_{k}$ the sample tree for the set of points $A_{k}$ (where $k$ is finite or $k=\infty$ ). Note that $\Sigma_{k}$ depends on the number $L$ and the coefficients $\lambda_{1} \cdots \lambda_{n}$. Clearly, for the sample tree one has

$$
\angle y_{2 j} y_{j} y_{2 j+1}=\angle y_{2 j} y_{j} y_{\lfloor j / 2\rfloor}=\angle y_{\lfloor j / 2\rfloor} y_{j} y_{2 j+1}=\frac{2 \pi}{3},
$$

Actually, by construction $y_{j}=F\left(y_{\lfloor j / 2\rfloor}, x_{2 j}, x_{2 j+1}\right)$ and all the angles of the triangle $\triangle x_{2 j} y_{\lfloor j / 2\rfloor} x_{2 j+1}$ do not exceed $2 \pi / 3$, and thus all the sides of the triangle are visible from $y_{j}$ at the angle $2 \pi / 3$.

It is easy to see that there is a $\bar{\lambda}>0$ such that if all $\lambda_{k}<\bar{\lambda}$, then all $\Sigma_{k}$ with $k$ finite and $k=\infty$ do not contain loops (homeomorphic images of $S^{1}$ ) and hence are topological trees (i.e. connected and locally connected compact sets without loops). Of course this condition must be satisfied for $\Sigma_{k}$ to be an optimal set.

3.1. Main result. The principal result of this paper is the following.

Theorem 3.1. Let $\Sigma_{k}$ (where $k$ is either finite or $k=\infty$ ) be the sample tree constructed with decreasing sequence of positive coefficients $\left\{\lambda_{j}\right\}$, satisfying

$$
\begin{aligned}
\lambda_{j} & \leq 1 / 5000, \quad j \geq 2, \\
120 \sum_{j=1}^{\infty} \lambda_{j} & <\pi / 42 .
\end{aligned}
$$

Then $\Sigma_{k} \in \mathcal{M}\left(\left\{y_{0}\right\} \cup A_{k}\right)$ and for every $S \in \mathcal{M}\left(\left\{y_{0}\right\} \cup A_{k}\right)$ one has $S_{k} \subset S \subset \Sigma_{k}$. 


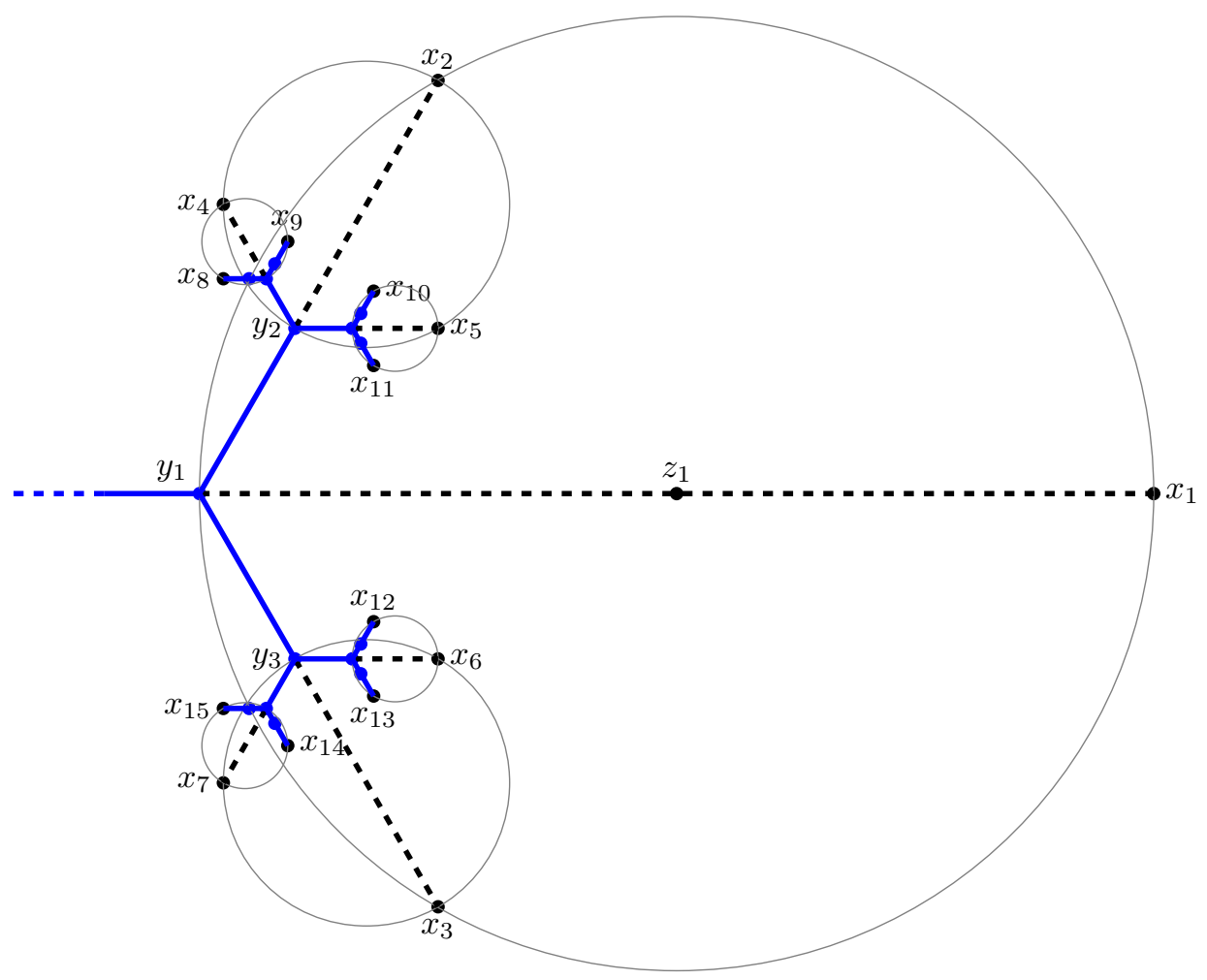

Figure 2. Three iterations in the construction of $\Sigma_{n}$. The set $\Sigma_{3}$ is blue.

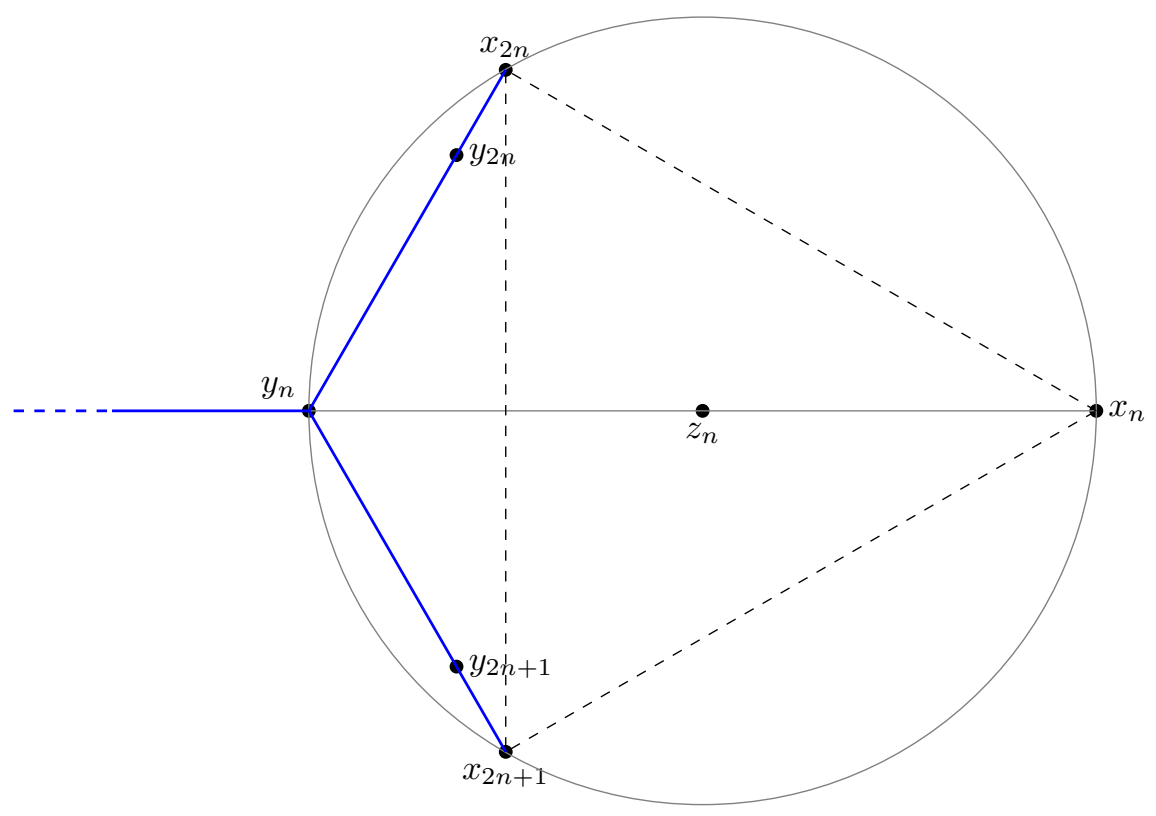

Figure 3. The iteration in the construction of $\Sigma_{n}$.

It is worth remarking that the proof of the above theorem gives a bit more, namely some stability of the result with precise geometric conditions on the data that guarantee still the same structure and length of the Steiner tree as well as its uniqueness. We stress however the requirement that the sequence $\left\{\lambda_{j}\right\}$ be vanishing 
as $j \rightarrow \infty$. It is not clear whether the similar statement is true for non vanishing sequences, say, for constant ones $\lambda_{j}=\lambda$ for some sufficiently small $\lambda>0$.

3.2. Some properties of the construction. For the readers' convenience we remark here the basic properties of our construction.

Recall that a $c \in \Sigma$ is called a topological endpoint of a compact connected metric space $\Sigma$, if for every $\varepsilon>0$ there is an open neighborhood $U$ of $c$ in $\Sigma$ with diam $U \leq \varepsilon$ and $\partial U$ being a singleton [3]. It is easy to note then that the closed set $A_{k}$ is made of the topological endpoints of the tree $\Sigma_{k}$ (for both $k$ finite and $k=+\infty$ ), and in the case $k=+\infty$ it is uncountable (this is immediate by identifying each $c \in A_{\infty}$ with the itinerary in the binary tree, say, encoded by a sequence of 0 and 1 standing for the directions chosen at each branching point). Further, it is totally disconnected (i.e. its connected components are singletons), which can of course be worked out "by hand" in our construction even for the case we do not know that $\Sigma_{k}$ are Steiner trees, but it is curious to observe that once we know $\Sigma_{k}$ to be a Steiner tree, the respective properties of $A_{k}$ can also be seen as a general property of endpoints of Steiner trees according to the following statement.

Proposition 3.2. Suppose that $\Sigma \subset E$ is a closed set solving the Steiner problem for some compact $C \subset E$, i.e. $\Sigma \in \mathcal{M}(C)$, where $E$ is a complete metric space, $\mathcal{H}^{1}(\Sigma)<+\infty$. If the set of endpoints $A$ of all connected components of $\Sigma$ is closed, then it is compact and totally disconnected. Moreover, $\Sigma \in \mathcal{M}(A)$.

Proof. Suppose that $A$ is closed. By theorem 7.6 from [5] one has $A \subset C$, hence it is compact. Further, if there is a shorter $\Sigma$ connecting $A$, then it also connects $C$, hence, $\Sigma \in \mathcal{M}(A)$. Again by theorem 7.6 from [5] one has that each connected component of $\Sigma$ has at most one endpoint at each connected component of $A$, hence, $A$ is totally disconnected.

Note that in our construction the set $A_{\infty}$ is compact, totally disconnected and perfect (i.e. it has no isolated points, say, by Lemma 3.5 which implies in particular that for each $c \in A_{\infty}$ there is an arbitrarily small ball containing $c$ and containing infinitely many other points of $A_{\infty}$ ), and hence it is homeomorphic to the Cantor set (which is yet another way to observe that it is uncountable).

Another easy statement below shows in particular that no binary tree can be an indecomposable Steiner tree for the compact set of its endpoints $A$, if $\mathcal{H}^{1}(A) \neq 0$. For simplicity it is provided here for sets in a Euclidean space although it is clearly valid in a much more general context, as easily can be deduced from the proof.

Proposition 3.3. Suppose that $\Sigma \subset \mathbb{R}^{n}$ is a compact connected set, and $\mathcal{H}^{1}(\Sigma)<$ $+\infty$. Then for its set of endpoints $A$ one has $\mathcal{H}^{1}(A)=0$.

Proof. Recall that if $\Sigma$ is a compact connected set with $\mathcal{H}^{1}(\Sigma)<+\infty$, then it is a trace of some Lipschitz curve $\theta$ of finite length which without loss of generality may be arclength parameterized over some interval $[0, l]$, so that $\theta([0, l])=\Sigma$. Let $c \in A$. Then for each $t \in(0, l)$ such that $\theta(t)=c$ one has $\dot{\theta}(t)$ either does not exist or $\dot{\theta}(t)=0$. In fact, otherwise there are two curves with traces $\Gamma_{1}=\theta([t, t+\varepsilon]) \subset \Sigma$ and $\Gamma_{2}=\theta([t-\varepsilon, t]) \subset \Sigma$ for some $\varepsilon>0$ such that $\Gamma_{1} \cap \Gamma_{2}=\{c\}$, and hence $c$ is not an endpoint of $\Sigma$. Thus $\{t \in[0, l]: \theta(t) \in A\}$ must have zero Lebesgue measure, which implies $\mathcal{H}^{1}(A)=0$.

In the sequel we also will need the following almost immediate technical lemmata.

Lemma 3.4. Every point $c \in A_{\infty}$ is a limit point of some sequence of points $a_{k} \in A_{k}$.

Proof. Consider an arbitrary point $c \in A_{\infty}=\Sigma_{\infty} \backslash S_{\infty}$. There is a subsequence $c_{k} \in S_{\infty}=\bigcup_{k=1}^{\infty} \sigma_{k}$ converging to $c$. It may be assumed without loss of generality 
that $c_{k} \in \sigma_{k} \backslash \sigma_{k-1}$ (otherwise, there would be an $n \in \mathbb{N}$ such that $c \in \bar{\sigma}_{n} \subset \sigma_{n+1} \subset$ $\left.S_{\infty}\right)$. Then $\operatorname{dist}\left(c_{k}, A_{k}\right) \leq \lambda_{k} \ldots \lambda_{1} L \rightarrow 0$. Since each $A_{k}$ is a compact set, there is $a_{k} \in A_{k}$ attaining this distance. But then

$$
\left|c-a_{k}\right| \leq\left|c-c_{k}\right|+\left|c_{k}-a_{k}\right| \rightarrow 0
$$

as $k \rightarrow \infty$.

Lemma 3.5. For the sample tree constructed with decreasing sequence of coefficients $\left\{\lambda_{k}\right\}$ satisfying $\lambda_{1}<1 / 2$ one has $\operatorname{Gen}\left(x_{n}\right) \subset B_{4 L \lambda_{1} \ldots \lambda_{g(n)}}\left(x_{n}\right)$ for every $n \in \mathbb{N}$.

Proof. Let $x_{p} \in \operatorname{Gen}\left(x_{n}\right)$. Then

$$
\operatorname{dist}\left(x_{n}, x_{p}\right) \leq \operatorname{dist}\left(y_{n}, x_{n}\right)+\operatorname{dist}\left(y_{n}, x_{p}\right) .
$$

We estimate dist $\left(y_{n}, x_{p}\right)$, keeping in mind that the radius of the circle circumscribed around the equilateral triangle $\triangle x_{2 j} x_{2 j+1} x_{j}$ is equal to $\lambda_{g(j)} \ldots \lambda_{1} L=\left|y_{j} x_{2 j}\right|=$ $\left|y_{j} x_{2 j+1}\right|$ (note that $F\left(x_{2 j}, x_{2 j+1}, x_{j}\right) \in\left[y_{j} x_{j}\right]$ is the center of this circle), and hence

$$
\operatorname{dist}\left(y_{\lfloor j / 2\rfloor}, y_{j}\right)<\operatorname{dist}\left(y_{\lfloor j / 2\rfloor}, x_{j}\right)=\lambda_{g(j)-1} \ldots \lambda_{1} L \text {. }
$$

One has then

$$
\begin{aligned}
\operatorname{dist}\left(y_{n}, x_{p}\right) & \leq \operatorname{dist}\left(y_{\lfloor p / 2\rfloor}, x_{p}\right)+\sum_{l=1}^{g(p)-g(n)-1} \operatorname{dist}\left(y_{\left\lfloor p / 2^{l}\right\rfloor}, y_{\left\lfloor p / 2^{l+1}\right\rfloor}\right) \\
& \leq L \lambda_{1} \ldots \lambda_{g(p)-1}+\sum_{l=1}^{g(p)-g(n)-1} L \lambda_{1} \ldots \lambda_{l} \\
& =\sum_{l=g(n)}^{g(p)-1} L \lambda_{1} \ldots \lambda_{l}=L \lambda_{1} \ldots \lambda_{g(n)}\left(1+\sum_{l=g(n)+1}^{g(p)-1} \lambda_{g(n)+1} \ldots \lambda_{l}\right) \\
& \leq L \lambda_{1} \ldots \lambda_{g(n)} \sum_{l=0}^{g(p)-g(n)-1} \lambda_{g(n)+1}^{l} \\
& \leq L \lambda_{1} \ldots \lambda_{g(n)} \frac{1-\lambda_{g(n)+1}^{g(p)-g(n)}}{1-\lambda_{g(n)+1}}<2 L \lambda_{1} \ldots \lambda_{g(n)},
\end{aligned}
$$

because

$$
\frac{1-\lambda_{g(n)+1}^{m-g(n)}}{1-\lambda_{g(n)+1}}<\frac{1}{1-\lambda_{g(n)+1}}<2,
$$

since $\lambda_{g(n)+1}<1 / 2$. But

$$
\text { dist }\left(y_{n}, x_{n}\right)=2 L \lambda_{1} \ldots \lambda_{g(n)},
$$

and therefore, $\operatorname{dist}\left(x_{p}, x_{n}\right)<4 L \lambda_{1} \ldots \lambda_{g(n)}$.

\section{Proof of the MAIN RESUlt}

Proof of Therem 3.1. The proof will be achieved in several steps. Namely,

- in Step 1 we will prove that any Steiner tree in $\mathcal{M}\left(\left\{y_{0}^{\prime}\right\} \cup A_{k}\right)$ has the structure similar to that of the sample tree, i.e. is still a binary tree with each bifurcation at the angle of $2 \pi / 3$ (we will call such a structure regular), once its root $y_{0}^{\prime}$ is not too far away from $y_{0}$ (the precise geometrical condition for "being not too far away" will be provided, and it is worth emphasizing that it allows the distance $\left|y_{0} y_{0}^{\prime}\right|$ to be arbitrarily large). This is the crucial step of the proof, and it will be accomplished by an inductive application of Lemma A.6. The latter is of certain interest itself: it deals with a Steiner set connecting a vertex of an equilateral triangle with two very small arbitrary 
compact sets very close to the other two vertices of this triangle, and says when it remains a tripod (like the Steiner tree connecting the vertices of this triangle) away from these sets, giving the answer in terms of a quantitative estimate on the data;

- in Step 2 we first show under the same hypothesis that every finite tree with regular structure connecting $\left\{y_{0}^{\prime}\right\} \cup A_{k}, k \in \mathbb{N}$, has length $\left|y_{0}^{\prime} x_{1}\right|$, which together with the result of the Step 1 proves that every finite Steiner tree in $\mathcal{M}\left(\left\{y_{0}^{\prime}\right\} \cup A_{k}\right)$ has length $\left|y_{0}^{\prime} x_{1}\right|$, so that that in particular the optimality of sample trees follows for the case $k$ finite. This is accomplished by a more or less straightforward application of Melzak's construction [2];

- then (still in Step 2) knowing the exact value of the length of Steiner trees $\Sigma_{k}^{\prime} \in \mathcal{M}\left(\left\{y_{0}^{\prime}\right\} \cup A_{k}\right)$ for $k$ finite, we show that it remains the same (i.e. equal to $\left.\left|y_{0}^{\prime} x_{1}\right|\right)$ also for $k=+\infty$, proving in particular also the optimality of the sample tree for this case. This is an almost immediate application of Gołąb theorem on semicontinuity of length along sequences of Hausdorff convergent connected compact sets, and of the optimality of $\Sigma_{k}^{\prime}$;

- and, finally, on Step 3 we will prove the uniqueness of the Steiner tree in $\mathcal{M}\left(\left\{y_{0}\right\} \cup A_{k}\right)$ (i.e. with root $\left.y_{0}\right)$. The latter will be done for both the cases $k$ finite and $k=+\infty$ simultaneously by the same argument (without distinguishing between these cases). Note that in fact, here only the case $k=+\infty$ is really interesting, since uniqueness for $k$ finite is well-known and follows from convexity of the length of a Steiner tree as a function of coordinates of branching points. However, the argument we use here works for both cases. Namely, we prove by induction that for any Steiner tree in $\mathcal{M}\left(\left\{y_{0}\right\} \cup A_{k}\right)$ its branching points coincide with those of the sample tree, which is done again with the help of Step 1 and Step 2.

Since the proof is quite lengthy and technical, we found it reasonable to put all the necessary auxiliary statements including the crucial Lemma A.6 in the Appendix. Step 1. We prove that an arbitrary Steiner tree $\Sigma_{k}^{\prime} \in \mathcal{M}\left(\left\{y_{0}^{\prime}\right\} \cup A_{k}\right)$ has the same structure of the sample tree (we will call it regular), if its root $y_{0}^{\prime}$ is not too far away from $y_{0}$. Namely, a tree with $2^{k}-1$ branching points $y_{j}^{\prime}$ and endpoints $\left\{x_{j}^{\prime}\right\}$, i.e.

$$
\Sigma_{k}^{\prime}=\sigma_{k}^{\prime} \cup \bigcup_{j=2^{k}}^{2^{k+1}-1}\left[y_{j}^{\prime}, x_{j}^{\prime}\right]
$$

where

$$
\sigma_{k}^{\prime}:=\left[y_{0}^{\prime}, y_{1}^{\prime}\right] \cup \bigcup_{j=1}^{2^{k+1}}\left(\left[y_{j}^{\prime}, y_{2 j}^{\prime}\right] \cup\left[y_{j}^{\prime}, y_{2 j+1}^{\prime}\right]\right)
$$

and

so that all the angles

$$
y_{j}^{\prime}=F\left(y_{2 j}^{\prime}, y_{\lfloor j / 2\rfloor}^{\prime}, y_{2 j+1}^{\prime}\right), \quad j=1, \ldots, 2^{k-2},
$$

$$
\angle y_{2 j}^{\prime} y_{j}^{\prime} y_{2 j+1}^{\prime}=\angle y_{2 j}^{\prime} y_{j}^{\prime} y_{\lfloor j / 2\rfloor}^{\prime}=\angle y_{\lfloor j / 2\rfloor}^{\prime} y_{j}^{\prime} y_{2 j+1}^{\prime}=\frac{2 \pi}{3}
$$

and further,

$$
y_{j}^{\prime}=F\left(x_{2 j}^{\prime}, x_{2 j+1}^{\prime}, y_{\lfloor j / 2\rfloor}^{\prime}\right), \quad j=2^{k-2}, \ldots, 2^{k-1}
$$

and hence,

$$
\angle x_{2 j}^{\prime} y_{j}^{\prime} x_{2 j+1}^{\prime}=\angle x_{2 j} y_{j}^{\prime} y_{\lfloor j / 2\rfloor}^{\prime}=\angle y_{\lfloor j / 2\rfloor}^{\prime} y_{j}^{\prime} x_{2 j+1}^{\prime}=\frac{2 \pi}{3},
$$

will be said to have regular structure of $k$-th generation with $k$ finite (in particular, this is the case of the sample tree according to (1)). The condition on the root $y_{0}^{\prime}$ to be "not too far away" from $y_{0}$ will be considered (and this is important in 
the sequel) in the angular terms, namely, we assume that $\angle y_{0}^{\prime} x_{1} y_{0}<\beta_{1}$ and $y_{0}^{\prime}$ be outside of the ball $\bar{B}_{\left(40 \lambda_{2}+1\right) \lambda_{1} L}\left(z_{1}\right)$ (see Figure 1$)$.

The point $y_{j}^{\prime}$ will be called branching point of $i$-th generation, if $i=g(j)$. Define the sequence

$$
\beta_{0}:=\frac{\pi}{6}-\frac{\pi}{21}, \quad \beta_{i+1}:=\beta_{i}+120 \lambda_{i+1} .
$$

Then according to the assumption (3) of the main theorem, for each index $i$ the inequality

$$
\frac{\pi}{6}-\frac{\pi}{21}<\beta_{i}<\frac{\pi}{7}=\frac{\pi}{6}-\frac{\pi}{42}
$$

holds. By induction on the generation $i$ we prove now the following

Claim: for all finite $i=1, \ldots, k$, the Steiner tree $\Sigma_{k}^{\prime}$ outside of the union of the balls $\bigsqcup_{g(l)=i+1} B_{20 \lambda_{i+1} \ldots \lambda_{1} L}\left(x_{l}\right)$ is the tree of $i$-th generation having regular structure (i.e. it is the tree with regular structure having branching points of all generations up to the $i$-th generation), with root $y_{0}^{\prime}$ and the set of leafs $x_{l}^{\prime}$ belonging to the union of circumferences $\bigsqcup_{g(l)=i+1} \partial B_{20 \lambda_{i+1} \ldots \lambda_{1} L}\left(x_{l}\right)$, with a single leaf on each circumference; moreover, each branching point $y_{j}^{\prime}$ of $i$-th generation is located inside the angle of measure $2 \beta_{i+1}$ with bisector $\left[x_{2 j} y_{j}\right)$ and outside of the ball $\bar{B}_{\left(40 \lambda_{i+2} L+1\right) \lambda_{i+1} \ldots \lambda_{1} L}\left(z_{2 j}\right)$, as well as inside the angle of the same value $2 \beta_{i+1}$ with bisector $\left[x_{2 j+1} y_{j}\right)$ and outside of the ball $\bar{B}_{\left(40 \lambda_{i+2} L+1\right) \lambda_{i+1} \ldots \lambda_{1} L}\left(z_{2 j+1}\right)$.

Note that $k$ in the above Claim is not necessarily finite.

Base of induction: $i=1$. According to Lemma 3.5 the set $A_{k}$ is located inside the balls with radius $4 \lambda_{1} \lambda_{2} L$ centered at $x_{2}$ and $x_{3}$. Since for the points $T^{\prime}:=y_{0}^{\prime}, T:=y_{0}, V:=x_{2}, U:=x_{3}$ and the sets $A:=A_{k}, A_{V}:=\operatorname{Gen}_{k}\left(x_{2}\right)$, $A_{U}:=\operatorname{Gen}_{k}\left(x_{3}\right)$ conditions of Lemma A.6 are satisfied, then there are points $x_{2}^{\prime}:=V^{\prime} \in \partial B_{20 \lambda_{2} \lambda_{1} L}\left(x_{2}\right)$ and $x_{3}^{\prime}:=U^{\prime} \in \partial B_{20 \lambda_{2} \lambda_{1} L}\left(x_{3}\right)$, such that outside of the balls $B_{20 \lambda_{2} \lambda_{1} L}\left(x_{2}\right)$ and $B_{20 \lambda_{2} \lambda_{1} L}\left(x_{3}\right)$ the optimal tree for $\left\{y_{0}^{\prime}\right\} \cup A_{k}$ coincides with the tripod connecting $y_{0}^{\prime}, U^{\prime}, V^{\prime}$ and having branching point $y_{1}^{\prime}=F\left(T^{\prime}, U^{\prime}, V^{\prime}\right)$. Since $T, T^{\prime}, U^{\prime}, V^{\prime}, W:=x_{1}$ satisfy the conditions of Lemma A.9 with $\beta:=\beta_{2}$ and $\alpha:=\angle T^{\prime} W T<\beta_{1}=\beta_{2}-120 \lambda_{2}$, then $y_{1}^{\prime}$ is located inside the angle of $2 \beta_{2}$ with bisector $\left[x_{2} y_{1}\right)$, while in view of Remark A.10 it does not belong to $\bar{B}_{\left(40 \lambda_{3} L+1\right) \lambda_{2} \lambda_{1} L}\left(z_{2}\right)$ (while applying Remark A.10 it is worth noting that here $O_{V}=z_{2}$ ).

The symmetrical assertion is also true: the point $y_{1}^{\prime}$ is inside the angle of $2 \beta_{2}$ with the bisector $\left[x_{3} y_{1}\right)$ and outside of the ball $\bar{B}_{\left(40 \lambda_{3} L+1\right) \lambda_{2} \lambda_{1} L}\left(z_{3}\right)$. So the base of induction is proved.

Step of induction. Consider an arbitrary branching point $y_{j}^{\prime}$ of $i$-th generation (i.e. $g(j)=i$ ), for which the inductive assumption holds, in particular, $y_{j}^{\prime}$ exists and is located inside the angle of $2 \beta_{i+1}$ with bisector $\left[x_{2 j} y_{j}\right.$ ) (in other words, $\angle y_{j}^{\prime} x_{2 j} y_{j}<$ $\left.\beta_{i+1}\right)$ and outside of the ball $\bar{B}_{\left(40 \lambda_{i+2} L+1\right) \lambda_{i+1} \ldots \lambda_{1} L}\left(z_{2 j}\right)$. The inductive assumption implies that $\Sigma_{k}^{\prime}$ contains a subtree connecting $y_{j}^{\prime}$ with $\partial B_{20 \lambda_{i+1} \ldots \lambda_{1} L}\left(x_{2 j}\right)$ and therefore, since other parts of $\Sigma_{k}^{\prime}$ cannot intersect this circle, it also contains the subtree connecting $y_{j}^{\prime}$ with $\operatorname{Gen}_{k}\left(x_{2 j}\right) \subset B_{4 \lambda_{i+1} \ldots \lambda_{1} L}\left(x_{2 j}\right)$ and both subtrees are optimal. Then in view of Lemma 3.5

$$
\operatorname{Gen}_{k}\left(x_{2 j}\right)=\operatorname{Gen}_{k}\left(x_{4 j}\right) \sqcup \operatorname{Gen}_{k}\left(x_{4 j+1}\right) \subset B_{4 \lambda_{i+2} \ldots \lambda_{1} L}\left(x_{4 j}\right) \sqcup B_{4 \lambda_{i+2} \ldots \lambda_{1} L}\left(x_{4 j+1}\right),
$$

the assumptions of Lemma A.6 hold with $A_{V}:=\operatorname{Gen}_{k}\left(x_{4 j}\right)$ and $A_{U}:=\operatorname{Gen}_{k}\left(x_{4 j+1}\right)$ ), and thus there exists a $V^{\prime} \in \partial B_{20 \lambda_{i+2} \ldots \lambda_{1} L}\left(x_{4 j}\right)$ and an $U^{\prime} \in \partial B_{20 \lambda_{i+2} \ldots \lambda_{1} L}\left(x_{4 j+1}\right)$ such that outside of the balls $B_{20 \lambda_{i+2} \ldots \lambda_{1} L}\left(x_{4 j}\right)$ and $B_{20 \lambda_{i+2} \ldots \lambda_{1} L}\left(x_{4 j+1}\right)$ any optimal set for $\left\{y_{j}^{\prime}\right\} \cup \operatorname{Gen}_{k}\left(x_{2 j}\right)$ coincides with the tripod connecting $y_{j}^{\prime}, U^{\prime}, V^{\prime}$ and having branching point $y_{2 j}^{\prime}=F\left(y_{j}^{\prime}, U^{\prime}, V^{\prime}\right)$. Then employing Lemma A.9 (with $T^{\prime}:=y_{j}^{\prime}$, $T:=y_{j}, V:=x_{4 j}, U=x_{4 j+1}, W:=x_{2 j}, \beta:=\beta_{i+2}, \alpha:=\angle T^{\prime} W T<\beta_{i+1}=$ $\beta_{i+2}-120 \lambda_{i+2}$ and $L \lambda_{1} \ldots \lambda_{i}$ in place of $L, \lambda_{i+1}$ in place of $\lambda_{1}$ and $\lambda_{i+2}$ in place of 
$\lambda_{2}$ ), we get that $y_{2 j}^{\prime}$ is inside the angle of $2 \beta_{i+2}$ with bisector $\left[x_{4 j} y_{2 j}\right)$ and outside of the ball $\bar{B}_{\left(40 \lambda_{i+3} L+1\right) \lambda_{i+2} \ldots \lambda_{1} L}\left(z_{4 j}\right)$. Moreover, the point $y_{2 j}^{\prime}$ is inside the angle of $2 \beta_{i+2}$ with bisector $\left[x_{4 j+1} y_{2 j}\right.$ ) and in view of Remark A.10 (used with the same notations) is outside of the ball $\bar{B}_{\left(40 \lambda_{i+3} L+1\right) \lambda_{i+2} \ldots \lambda_{1} L}\left(z_{4 j+1}\right)$. Similarly, the point $y_{2 j+1}^{\prime}$ is inside the angle of $2 \beta_{i+2}$ with bisector $\left[x_{4 j+2} y_{2 j+1}\right)$ and outside of the ball $\bar{B}_{\left(40 \lambda_{i+3} L+1\right) \lambda_{i+2} \ldots \lambda_{1} L}\left(z_{4 j+2}\right)$. Moreover, it is inside the angle of $2 \beta_{i+2}$ with bisector $\left[x_{4 j+3} y_{2 j+1}\right)$ and outside of the ball $\bar{B}_{\left(40 \lambda_{i+3} L+1\right) \lambda_{i+2} \ldots \lambda_{1} L}\left(z_{4 j+3}\right)$.

Further, since $y_{2 j}^{\prime}=F\left(y_{j}, U^{\prime}, V^{\prime}\right)$, one has

$$
\angle x_{4 j}^{\prime} y_{2 j}^{\prime} x_{4 j+1}^{\prime}=\angle y_{j}^{\prime} y_{2 j}^{\prime} x_{4 j}^{\prime}=\angle x_{4 j+1}^{\prime} y_{2 j}^{\prime} y_{j}^{\prime}=\frac{2 \pi}{3}
$$

for $x_{4 j+1}^{\prime}:=U^{\prime}, x_{4 j}^{\prime}:=V^{\prime}$. The analogous statement is true for the point $y_{2 j+1}^{\prime}$. Thus the Steiner tree $\Sigma_{k}^{\prime}$ outside of the balls $\bigsqcup_{g(l)=i+2} B_{20 \lambda_{i+2} \ldots \lambda_{1} L}\left(x_{l}\right)$ coincides with the tree of the $i$-th generation with the regular structure which connects the root $y_{0}^{\prime}$ with some vertices from the circumferences $\bigsqcup_{g(l)=i+2} \partial B_{20 \lambda_{i+2} \ldots \lambda_{1} L}\left(x_{l}\right)$ (one vertex on each of the circumferences), which concludes the proof of the step of induction.

Step 2. We prove that the length of any Steiner tree for $\left\{y_{0}^{\prime}\right\} \cup A_{k}$ (with $k$ finite or infinite) is equal to $\left|y_{0}^{\prime} x_{1}\right|$, once one has, as assumed on Step 1 , that $\angle y_{0} x_{1} y_{0}^{\prime}<\beta_{1}$ and the point $y_{0}^{\prime}$ is outside of the ball $\bar{B}_{\left(1+40 \lambda_{2}\right) \lambda_{1} L}\left(z_{1}\right)$.

First assume $k$ to be finite. We will show the claim for every tree with regular structure connecting the set of points $\left\{y_{0}^{\prime}\right\} \cup A_{n}$. For this purpose we again use induction. The base $(i=0)$ is obvious, since every tree having the regular structure connecting the points of the set $\left\{y_{0}^{\prime}\right\} \cup A_{0}=\left\{y_{0}^{\prime}, x_{1}\right\}$ is just a segment with length $\left|y_{0}^{\prime} x_{1}\right|$. It suffices thus to prove that every tree of the regular structure connecting the vertices of the set $y_{0}^{\prime} \cup A_{n}$ has length $\left|y_{0}^{\prime} x_{1}\right|$, if the same is true for all trees of the regular structure connecting the vertices of the set $y_{0}^{\prime} \cup A_{n-1}$. To this aim assume that $p$ is such that $g(p)=n$. Then

$$
y_{p}^{\prime}=F\left(x_{2 p}, x_{2 p+1}, y_{\lfloor p / 2\rfloor}\right) .
$$

By corollary to Ptolemy's theorem (Lemma A.1) if $y_{p}^{\prime}$ is on the circle circumscribed around the triangle $\triangle x_{2 p} x_{2 p+1} x_{p}$ and $\angle x_{2 p} y_{p}^{\prime} x_{2 p+1}=\frac{2 \pi}{3}$ (which is true in view of (4)), then $\left|y_{p}^{\prime} x_{2 p}\right|+\left|y_{p}^{\prime} x_{2 p+1}\right|=\left|y_{p}^{\prime} x_{p}\right|$. Let us consider an arbitrary tree of regular structure connecting the vertices of the set $y_{0}^{\prime} \cup A_{n}$, namely,

$$
\Sigma_{n}^{\prime}=\sigma_{n}^{\prime} \cup \bigcup_{j=2^{n}}^{2^{n+1}}\left[y_{j}^{\prime}, x_{j}\right]=\left[y_{0}^{\prime}, y_{1}^{\prime}\right] \cup \bigcup_{j=1}^{2^{n+1}}\left[y_{j}^{\prime}, y_{2 j}^{\prime}\right] \cup\left[y_{j}^{\prime}, y_{2 j+1}^{\prime}\right] \cup \bigcup_{j=2^{n}}^{2^{n+1}-1}\left[y_{j}^{\prime}, x_{j}\right]
$$

Denote

$$
\begin{aligned}
& \sigma_{n-1}^{\prime}:=\left[y_{0}^{\prime}, y_{1}^{\prime}\right] \cup \bigcup_{j=1}^{2^{n}}\left[y_{j}^{\prime}, y_{2 j}^{\prime}\right] \cup\left[y_{j}^{\prime}, y_{2 j+1}^{\prime}\right], \\
& \Sigma_{n-1}^{\prime}:=\sigma_{n-1}^{\prime} \cup \bigcup_{j=2^{n-1}}^{2^{n}-1}\left[y_{j}^{\prime}, x_{j}\right] .
\end{aligned}
$$

Since the tree $\Sigma_{n}^{\prime}$ has the regular structure, obviously, $\Sigma_{n-1}^{\prime}$ has a regular structure too, since there are no new branching points: with $g(j)=n$ one has

$$
\angle y_{\lfloor j / 2\rfloor} y_{j}^{\prime} x_{j}=\angle y_{\lfloor j / 2\rfloor} y_{j}^{\prime} y_{2 j}^{\prime}+\angle y_{2 j}^{\prime} y_{j}^{\prime} x_{j}=\frac{2 \pi}{3}+\frac{\pi}{3}=\pi,
$$

The second equality in the above chain is true because of the regular structure of $\Sigma_{n}^{\prime}$. Since $\Sigma_{n-1}^{\prime}$ has the regular structure, we can apply the induction hypothesis:

$$
\mathcal{H}^{1}\left(\Sigma_{n-1}^{\prime}\right)=\left|y_{0}^{\prime} x_{1}\right| \text {. }
$$


To verify the step of induction we only have to prove that

$$
\mathcal{H}^{1}\left(\Sigma_{n-1}^{\prime}\right)=\mathcal{H}^{1}\left(\Sigma_{n}^{\prime}\right)
$$

It is enough to verify that

$$
\mathcal{H}^{1}\left(\bigcup_{j=2^{n-1}}^{2^{n}-1}\left[y_{j}^{\prime}, x_{j}\right]\right)=\mathcal{H}^{1}\left(\bigcup_{j=2^{n-2}+1}^{2^{n-1}}\left[y_{j}^{\prime}, y_{2 j}^{\prime}\right] \cup\left[y_{j}^{\prime}, y_{2 j+1}^{\prime}\right] \cup \bigcup_{j=2^{n}}^{2^{n+1}-1}\left[y_{j}^{\prime}, x_{j}\right]\right) .
$$

The latter equation is true because for $p$ satisfying $g(p)=n-1$ one has

$$
\begin{aligned}
\left|y_{p}^{\prime} x_{p}\right| & =\left|y_{p}^{\prime} x_{2 p}\right|+\left|y_{p}^{\prime} x_{2 p+1}\right| \quad \text { in view of Lemma A.1 } \\
& =\left|y_{p}^{\prime} y_{2 p}^{\prime}\right|+\left|y_{2 p}^{\prime} x_{2 p}\right|+\left|y_{p}^{\prime} y_{2 p+1}^{\prime}\right|+\left|y_{2 p+1}^{\prime} x_{2 p+1}\right| \quad \text { in view of (5). }
\end{aligned}
$$

Therefore it is proven that every (finite) tree with the regular structure has length $\left|y_{0}^{\prime} x_{1}\right|$. Since in Step 1 it has been proven that every Steiner tree has regular structure, we have that the length of every optimal tree for the set $\left\{y_{0}^{\prime}\right\} \cup A_{k}$ is $\left|y_{0}^{\prime} x_{1}\right|$ for all finite $k$.

Let us now consider a case of the infinite number of vertices $(k=\infty)$. We will find the length of the tree $\Sigma_{\infty}^{\prime} \in \mathcal{M}\left(\left\{y_{0}^{\prime}\right\} \cup A_{\infty}\right)$. It cannot be greater than $\left|y_{0}^{\prime} x_{1}\right|$, because, in view of Lemma 3.4, the limit in Hausdorff distance $\tilde{\Sigma}_{\infty}$ of each subsequence of trees having the regular structure $\Sigma_{k}^{\prime}$ connects the points of the set $\left\{y_{0}^{\prime}\right\} \cup A_{\infty}\left(\right.$ as $\left.A_{k} \subset \Sigma_{k}^{\prime}\right)$ and its length satisfies

$$
\mathcal{H}^{1}\left(\tilde{\Sigma}_{\infty}\right) \leq \liminf _{n \rightarrow \infty} \mathcal{H}^{1}\left(\Sigma_{n}^{\prime}\right)=\left|y_{0}^{\prime} x_{1}\right|,
$$

because of Gołąb theorem on lower semicontinuity of $\mathcal{H}^{1}$ over Hausdorff convergent sequence of connected compact sets (theorem 3.3 from [5]). We prove now by contradiction that the length of $\Sigma_{\infty}$ cannot be less than $\left|y_{0}^{\prime} x_{1}\right|$. Assume the contrary, i.e. that it is $\left|y_{0}^{\prime} x_{1}\right|-\varepsilon$, where $\varepsilon>0$. Estimating the length of the set

$$
R_{n}:=\bigcup_{k=n}^{\infty} \bigcup_{j=2^{k-1}}^{2^{k}-1}\left(\left[x_{j} z_{j}\right] \cup\left[x_{2 j} z_{j}\right] \cup\left[x_{2 j+1} z_{j}\right]\right)
$$

connecting the vertices of $A_{n}$ and $A_{\infty}$ (note that it is the union of sets connecting the vertices of the generations $k-1$ and $k$, with $k \geq n)$, we get

$$
\begin{aligned}
\mathcal{H}^{1}\left(R_{n}\right) & =\sum_{k=n}^{\infty} \sum_{n=2^{k-1}}^{2^{k}-1}\left|x_{j} z_{j}\right|+\left|x_{2 n} z_{j}\right|+\left|x_{2 n+1} z_{j}\right|=\sum_{k=n}^{\infty} \sum_{n=2^{k-1}}^{2^{k}-1} 3 \lambda_{1} \ldots \lambda_{k} L \\
& \leq \sum_{k=n}^{\infty} 3 L\left(2 \lambda_{1}\right)^{k}=3 L \frac{\left(2 \lambda_{1}\right)^{n}}{1-2 \lambda_{1}} \rightarrow 0
\end{aligned}
$$

as $n \rightarrow \infty$. Choose an $n \in \mathbb{N}$ such that $\mathcal{H}^{1}\left(R_{n}\right)<\varepsilon / 2$. Then the points of the set $\left\{y_{0}^{\prime}\right\} \cup A_{n}$ with $n$ finite can be connected by $\Sigma_{\infty}^{\prime} \cup R_{n}$, namely, the points of the set $\left\{y_{0}^{\prime}\right\} \cup A_{\infty}$ are connected by $\Sigma_{\infty}^{\prime}$, and then points of the set $A_{n}$ are connected to points of the set $\left\{y_{0}^{\prime}\right\} \cup A_{\infty}$ by $R_{n}$. The total length of this construction does not exceed

$$
\left(\left|y_{0}^{\prime} x_{1}\right|-\varepsilon\right)+\frac{\varepsilon}{2}<\left|y_{0}^{\prime} x_{1}\right|
$$

which contradicts the fact that every optimal tree $\left\{y_{0}^{\prime}\right\} \cup A_{n}$ has length $\left|y_{0}^{\prime} x_{1}\right|$. This proves that every optimal tree for the set $\left\{y_{0}^{\prime}\right\} \cup A_{\infty}$ has length $\left|y_{0}^{\prime} x_{1}\right|$.

Step 3. We prove now uniqueness of the Steiner tree for $\left\{y_{0}\right\} \cup A_{k}$ with $k$ either finite or $k=\infty$ at once (without distinguishing the two cases). Since on Step 1 it has been already proven that every such tree $\Sigma_{k}^{\prime}$ has regular structure outside the respective balls, it suffices to show that all the branching points $y_{j}^{\prime}$ are at the sample position, i.e. $y_{j}^{\prime}=y_{j}$ for all $j \in \mathbb{N}$. We will do it by induction on the generation $i$. Since $y_{0}^{\prime}:=y_{0}$, base of induction is proven. Let us prove the inductive step. 
Let the claim be true for all branching points $y_{j}^{\prime}$ up to the $i$-th generation included $(g(j) \leq i)$. Then, as it was shown on Step $1, \Sigma_{k}^{\prime}$ has the regular structure outside the respective balls and hence

$$
\Sigma_{k}^{\prime}=\left[y_{0}, y_{1}\right] \cup \bigsqcup_{j=1}^{2^{i-1}}\left[y_{j}, y_{2 j}\right] \cup \bigsqcup_{j=2^{i-1}}^{2^{i}-1} \operatorname{Tr}_{j},
$$

where $\operatorname{Tr}_{j} \in \mathcal{M}\left(\left\{y_{j / 2}\right\} \cup \operatorname{Gen}_{k}\left(x_{j}\right)\right)$. For the length of $\Sigma_{k}^{\prime}$ to be minimum, it is necessary that the trees $\operatorname{Tr}_{l}$ have minimal length each, where $g(l)=i+1$. Without loss of generality, consider the structure of $\operatorname{Tr}_{2 j}$, where $g(j)=i$ and $y_{j}^{\prime}=y_{j}$ by the inductive hypothesis.

Then, using Step 1 with $y_{j}$ in place of $y_{0}, \operatorname{Gen}_{k}\left(x_{2 j}\right)$ in place of $A_{k}, k-i$ in place of $k, \lambda_{i} \ldots \lambda_{1} L$ in place of $L, x_{2^{g(l)} j+l-2^{g(l)}}$ in place of $x_{l}$ and $\lambda_{l+i}$ in place of $\lambda_{l}$, we get that outside of the balls $B_{20 \lambda_{i+2} \ldots \lambda_{1} L}\left(x_{4 j}\right)$ and $B_{20 \lambda_{i+2} \ldots \lambda_{1} L}\left(x_{4 j+1}\right)$ each tree $\operatorname{Tr}_{2 j}$, optimal for the set $\left\{y_{j}\right\} \cup \operatorname{Gen}_{k}\left(x_{2 j}\right)$, coincides with the tripod connecting $y_{j}, U^{\prime}$, $V^{\prime}$ and having branching point $y_{2 j}^{\prime}=F\left(y_{j}, U^{\prime}, V^{\prime}\right)$, where $V^{\prime} \in \partial B_{20 \lambda_{i+2} \ldots \lambda_{1} L}\left(x_{4 j}\right)$ and $U^{\prime} \in \partial B_{20 \lambda_{i+2} \ldots \lambda_{1} L}\left(x_{4 j+1}\right)$. Then $\operatorname{Tr}_{2 j}=\left[y_{j}, y_{2 j}^{\prime}\right] \sqcup \operatorname{Tr}_{4 j}^{\prime} \sqcup \operatorname{Tr}_{4 j+1}^{\prime}$, where $\operatorname{Tr}_{l}^{\prime} \in$ $\mathcal{M}\left(\left\{y_{l / 2}^{\prime}\right\} \cup \operatorname{Gen}_{k}\left(x_{l}\right)\right)$. Note that conditions of Lemma A.9 and Remark A.10 are fulfilled with $T^{\prime}:=T:=y_{j}, \beta:=\frac{\pi}{6}-\frac{\pi}{21}+120 \lambda_{i+1}$ and $\lambda_{i+1}$ in place of $\lambda_{2}$, because

$$
0=\angle T W T^{\prime}<\beta-120 \lambda_{i+1}=\frac{\pi}{6}-\frac{\pi}{21} .
$$

Thus the branching point $y_{2 j^{\prime}}$ is inside the angle of $2 \beta$ with the bisector $\left[x_{4 j+1} y_{2 j}\right)$ and outside of the ball $\bar{B}_{\left(40 \lambda_{i+3}+1\right) \lambda_{i+2} \ldots \lambda_{1} L}\left(z_{2 j}\right)$. Then the conditions of the Step 2 are satisfied for the point $y_{2 j}^{\prime}$ and the set $\operatorname{Gen}_{k}\left(x_{4 j}\right)$ (with $y_{2 j}$ and $y_{2 j}^{\prime}$ in place of $y_{0}$ and $y_{0}^{\prime}$, with $\operatorname{Gen}_{k}\left(x_{4 j}\right)$ in place of $A_{k}$, with $k-i-1$ in place of $k, \lambda_{i+1} \ldots \lambda_{1} L$ in place of $L, x_{2^{g(l)+1} j+l-2^{g(l)}}$ in place of $x_{l}, \lambda_{l+i+1}$ in place $\lambda_{l}$ and $\beta$ in place of $\left.\beta_{1}\right)$. We thus have that the length of the Steiner tree, connecting the points of the set $\left\{y_{2 j}^{\prime}\right\} \cup \operatorname{Gen}_{k}\left(x_{4 j}\right)$, is $\left|y_{2 j}^{\prime} x_{4 j}\right|$. So

$$
\mathcal{H}^{1}\left(\operatorname{Tr}_{4 j}^{\prime}\right)=\left|y_{2 j}^{\prime} x_{4 j}\right|
$$

Similarly,

Therefore,

$$
\mathcal{H}^{1}\left(\operatorname{Tr}_{4 j+1}^{\prime}\right)=\left|y_{2 j}^{\prime} x_{4 j+1}\right|
$$

$$
\mathcal{H}^{1}\left(\operatorname{Tr}_{2 j}\right)=\mathcal{H}^{1}\left(\left[y_{j}, y_{2 j}^{\prime}\right] \sqcup \operatorname{Tr}_{4 j}^{\prime} \sqcup \operatorname{Tr}_{4 j+1}^{\prime}\right)=\left|y_{j} y_{2 j}^{\prime}\right|+\left|y_{2 j}^{\prime} x_{4 j}\right|+\left|y_{2 j}^{\prime} x_{4 j+1}\right| .
$$

Since $\operatorname{Tr}_{2 j}$ has minimum possible length, then $y_{2 j}^{\prime}$ must minimize the expression on the righthand side, and hence coincide with the Fermat point $F\left(y_{j}, x_{4 j}, x_{4 j+1}\right)=$ $y_{2 j}$. Thus the induction step is proven, concluding therefore the proof of the claim of Step 3, and hence, the proof of the theorem.

\section{Appendix A. Auxiliary lemmata}

In this section we will provide some technical assertions. The most important results here which are of certain independent interest are Lemma A.3 and Lemma A.6.

To make the readers' life easier we recall the following very classical result the proof of which can be found in virtually any nice book on elementary geometry.

Lemma A.1. (corollary to Ptolemy's theorem) If the quadrilateral $A B C D$ is inscribed in a circumference and the angles $\angle B C D=\angle C B D=\pi / 3$, then $|A C|=$ $|A B|+|A D|$.

Consider now a triangle $\triangle T U V$ with all the angles less than $2 \pi / 3$. We will study what happens to its Fermat point, if the vertices of the triangle change their positions not too much, so that all angles remain less than $2 \pi / 3$. The quantitative answer to this question is given in Lemma A.3.

We start with the following notation. Denote 
- $Y:=F(T, U, V)$;

- $L:=|T Y|+|Y U|+|Y V|$.

Lemma A.2. Let $T$ be a point inside the angle $\angle U W V$ and outside of the closed circle circumscribed around the equilateral triangle $\triangle U W V$. Then the only compact $S \in \mathcal{M}(\{T, U, V\})$ is a tripod with branching in the Fermat point $F(T, U, V)$. The length $\mathcal{H}^{1}(S)=|T W|$ can be found from Ptolemy's theorem.

Proof. It suffices to observe that each angle of the triangle $\triangle T U V$ is less than $2 \pi / 3$ and so the respective Steiner tree is a tripod.

Assume now that $|T U|=|T V|$. We describe a simple construction drawn in Figure 4. Note that $R:=|Y U|=|Y V|$ is the radius of the circle circumscribed around the equilateral triangle $\triangle V U W$. Denote the center of this circle by $O$. Let further

- $\lambda_{1}:=R / L, \lambda_{1}<3 / 7, \lambda_{2}<1 / 120$

- the line $t$ be a common tangent to the circles $B_{\tau}(V)$ and $B_{\tau}(U)$ with $\tau:=$ $20 \lambda_{1} \lambda_{2} L$ so that these circles are on the opposite sides of it. Note that that under our assumption on $\lambda_{2}$ we have that the circles $\bar{B}_{\tau}(V)$ and $\bar{B}_{\tau}(U)$ are disjoint;

- $P_{V}:=t \cap \partial B_{\tau}(V), P_{U}:=t \cap \partial B_{\tau}(U)$. Without loss of generality assume that $\left|T P_{V}\right|<\left|T P_{U}\right|$;

- the point $P_{W}$ is such that the triangle $\triangle P_{V} P_{U} P_{W}$ is equilateral (there are two such points; we choose the one closest to $W$ );

- the ray $s_{V}$ starts from the point $W$ and is parallel to the line $\left(P_{V} P_{W}\right)$;

- the ray $s_{U}$ is symmetric to the ray $s_{V}$ with respect to the line $(T W)$.

We emphasize that in view of the requirements on $\lambda_{1}$ and $\lambda_{2}$ one has that the balls $\bar{B}_{\tau}(U), \bar{B}_{\tau}(V)$ and $\bar{B}_{2 \tau}(W)$ are disjoint, and the point $T$ is outside of the ball $\bar{B}_{R+2 \tau}(O)$ as can be verified by an elementary calculation.

Lemma A.3. The following assertions hold true.

(i) $\psi:=\angle\left(s_{V},(T W)\right)=\arccos \frac{40}{\sqrt{3}} \lambda_{2}-\frac{\pi}{3}$, so that $\psi \rightarrow \frac{\pi}{6}$ as $\lambda_{2} \rightarrow 0$.

(ii) If the point $T^{\prime}$ is such that $\angle T W T^{\prime}<\psi$, then

$$
\left[T T^{\prime}\right] \cap s_{V}=\left[T T^{\prime}\right] \cap s_{U}=\emptyset .
$$

(iii) If $T^{\prime}$ is such that

$$
\left[T^{\prime} T\right] \cap s_{U}=\left[T^{\prime} T\right] \cap s_{V}=\emptyset
$$

(in view of assertion (ii) it is true when $\angle T W T^{\prime}<\psi$ ) and $T^{\prime}$ is outside of the ball $\bar{B}_{R+2 \tau}(O)$, then for every $U^{\prime} \in \bar{B}_{\tau}(U)$ and $V^{\prime} \in \bar{B}_{\tau}(V)$ the unique Steiner tree $S^{\prime} \in \mathcal{M}\left(\left\{T^{\prime}, U^{\prime}, V^{\prime}\right\}\right)$ is a tripod with $\mathcal{H}^{1}\left(S^{\prime}\right)=\left|T^{\prime} W^{\prime}\right|$ whenever the triangle $\triangle V^{\prime} U^{\prime} W^{\prime}$ is equilateral.

Remark A.4. Under the assumptions of Lemma A.3, the set of possible positions of the point $W^{\prime}$ corresponding to all the possible $U^{\prime} \in \partial B_{\tau}(U)$ and $V^{\prime} \in \partial B_{\tau}(V)$, as well as to all the possible $U^{\prime} \in \bar{B}_{\tau}(U)$ and $V^{\prime} \in \bar{B}_{\tau}(V)$, is the closed ball $\bar{B}_{2 \tau}(W)$.

Indeed,

$$
\begin{aligned}
W & =V+\mathfrak{R}_{\frac{\pi}{3}}(U-V), \\
W^{\prime} & =V^{\prime}+\mathfrak{R}_{\frac{\pi}{3}}\left(U^{\prime}-V^{\prime}\right),
\end{aligned}
$$

where $\mathfrak{R}_{\frac{\pi}{3}} \in S O(2)$ is the matrix representing the rotation by $\pi / 3$, then

$$
\begin{aligned}
W^{\prime} & =V+\left(V^{\prime}-V\right)+\mathfrak{R}_{\frac{\pi}{3}}(U-V)+\mathfrak{R}_{\frac{\pi}{3}}\left(\left(U^{\prime}-U\right)-\left(V^{\prime}-V\right)\right) \\
& =W+\mathfrak{R}_{\frac{\pi}{3}}\left(U^{\prime}-U\right)+\left(I d-\mathfrak{R}_{\frac{\pi}{3}}\right)\left(V^{\prime}-V\right) .
\end{aligned}
$$




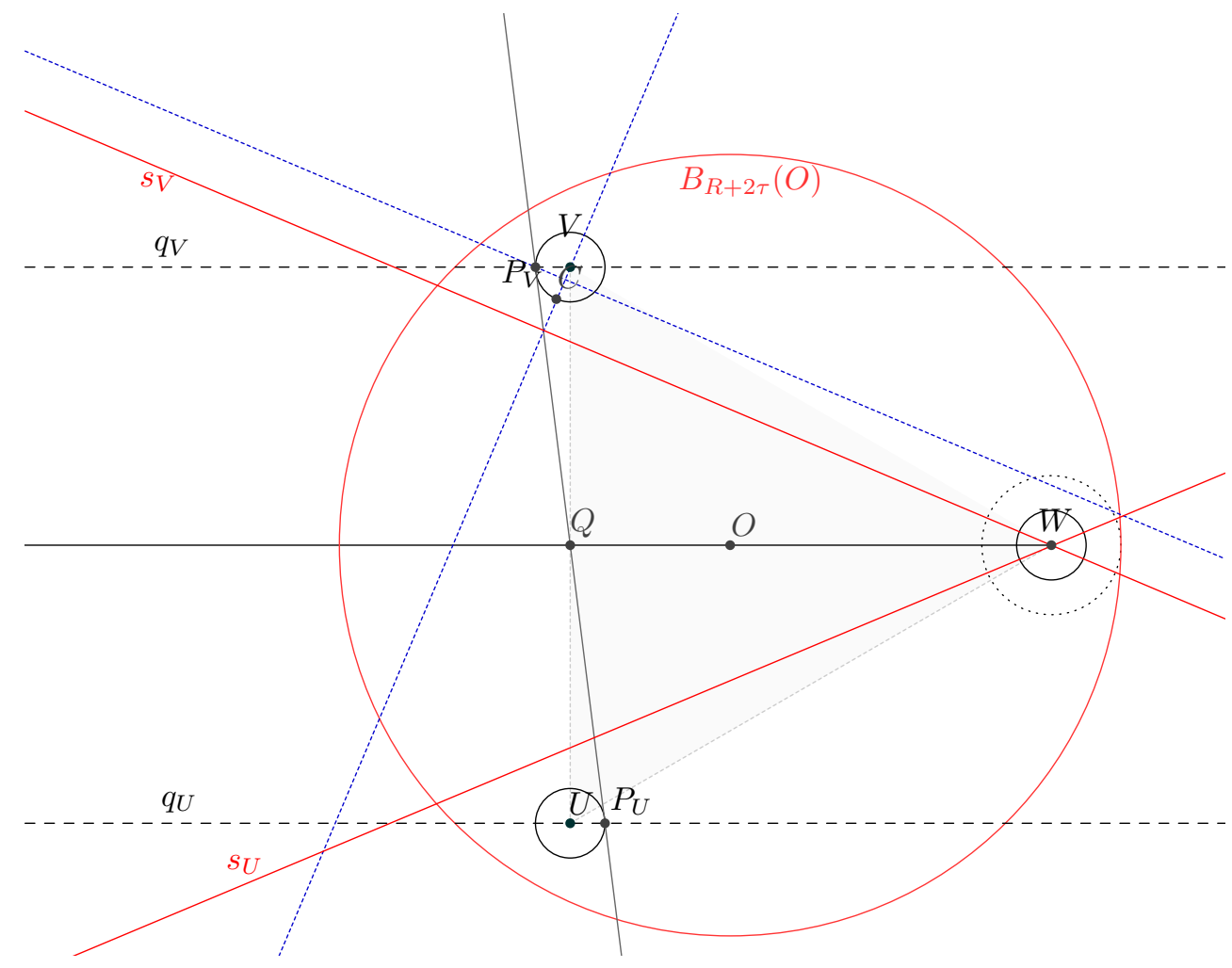

Figure 4. The construction used in Lemma A.3.

Therefore,

$$
\begin{aligned}
\left\{W^{\prime}: V^{\prime} \in \partial B_{\sigma}(V), U^{\prime} \in \partial B_{\rho}(U)\right\} & =W+\mathfrak{R}_{\frac{\pi}{3}}\left(\partial B_{\rho}(0)\right)+\left(I d-\mathfrak{R}_{\frac{\pi}{3}}\right)\left(\partial B_{\sigma}(0)\right) \\
& =W+\partial B_{\rho}(0)+\partial B_{\sigma}(0) \\
& =W+\bar{B}_{\rho+\sigma}(0) \backslash B_{(\rho-\sigma) \vee 0}(0) \\
& =\bar{B}_{\rho+\sigma}(W) \backslash B_{(\rho-\sigma) \vee 0}(W),
\end{aligned}
$$

where $B_{0}(0):=\emptyset$, the second equality is valid since

$$
\left(I d-\mathfrak{R}_{\frac{\pi}{3}}\right)\left(\partial B_{\sigma}(0)\right)=\partial B_{\sigma}(0) .
$$

Applying this assertion for $\sigma=\rho:=\tau$ as well as for all $\sigma \in[0, \tau], \rho \in[0, \tau]$, we get

$$
\left\{W^{\prime}: V^{\prime} \in \partial B_{\tau}(V), U^{\prime} \in \partial B_{\tau}(U)\right\}=\left\{W^{\prime}: V^{\prime} \in \bar{B}_{\tau}(V), U^{\prime} \in \bar{B}_{\tau}(U)\right\}=\bar{B}_{2 \tau}(W)
$$

as claimed.

Remark A.5. Under the assumptions of Lemma A.3, for the open ball $B^{\prime}$ circumscribed around the triangle $\triangle V^{\prime} W^{\prime} U^{\prime}$ one has

$$
\partial B^{\prime} \subset \bar{B}_{R+2 \tau}(O) \backslash B_{R-2 \tau}(O)
$$

so that

$$
B^{\prime} \subset B_{R+2 \tau}(O) .
$$

In fact, for every $z^{\prime} \in \partial B^{\prime}$ in the arc connecting $V^{\prime}$ and $W^{\prime}$, we have that there is a rotation $\mathfrak{R}$ by the angle not exceeding $\pi / 3$, such that $z^{\prime}=U^{\prime}+\mathfrak{R}\left(V^{\prime}-U^{\prime}\right)$. Denoting $z:=U+\mathfrak{R}(V-U)$, we have that clearly $z \in \partial B$ in the arc connecting $V$ and $W$, where $B$ is the circle circumscribed around $\triangle U W V$, and

$$
z^{\prime}-z=\mathfrak{R}\left(V^{\prime}-V\right)+(I d-\mathfrak{R})\left(U^{\prime}-U\right) .
$$




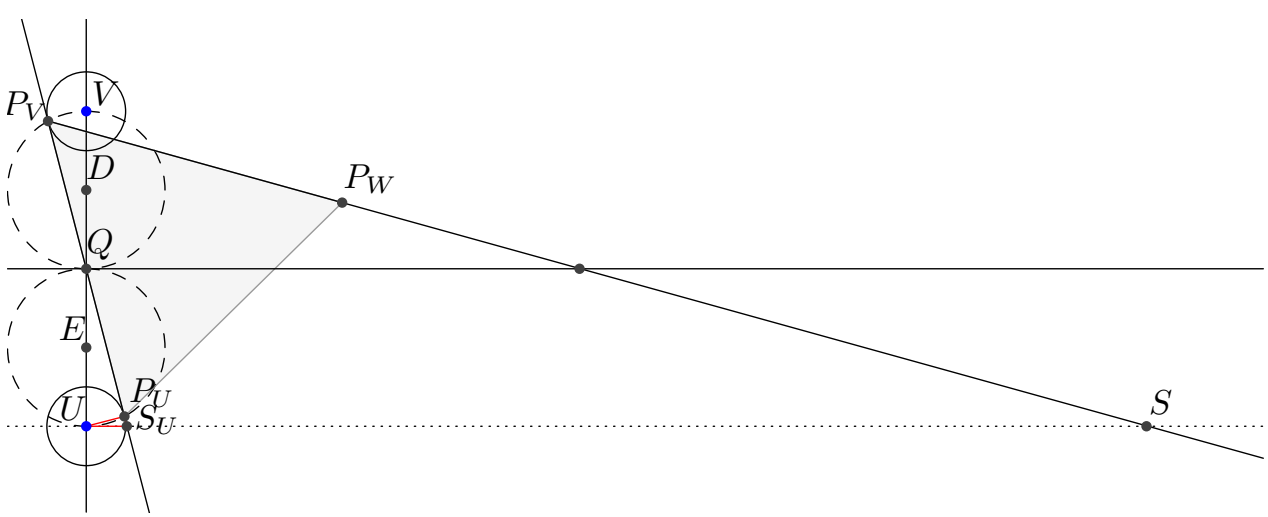

FiguRE 5. The construction used in the proof of Lemma A.3.

Letting $\sigma:=\left|V^{\prime}-V\right|$ and $\rho:=\left|U^{\prime}-U\right|$, we get that

$$
\begin{aligned}
z^{\prime}-z & \in \mathfrak{R}\left(\partial B_{\sigma}(0)\right)+(I d-\mathfrak{R})\left(\partial B_{\rho}(0)\right) \\
& \left.\subset \partial B_{\sigma}(0)\right)+\bar{B}_{\rho}(0) \subset \bar{B}_{\rho+\sigma}(0) \backslash B_{(\rho-\sigma) \vee 0}(0),
\end{aligned}
$$

the second inclusion being valid because the angle of the rotation does not exceed $\pi / 3$. Thus, minding that $\sigma \leq \tau$ and $\rho \leq \tau$, we get

$$
z^{\prime}-z \in \bar{B}_{2 \tau}(0) \text {. }
$$

Analogously, for every $z^{\prime} \in \partial B^{\prime}$ in the arc connecting $U^{\prime}$ and $W^{\prime}$, there is a $z \in \partial B$ in the arc connecting $U$ and $W$ such that (6) holds. In other words, the arc of $\partial B^{\prime}$ connecting $V^{\prime}$ and $W^{\prime}$ (resp. $U^{\prime}$ and $W^{\prime}$ ) belongs to the (closed) $2 \tau$-neighborhood of the arc of $\partial B$ connecting $V$ and $W$ (resp. $U$ and $W$ ). To show the analogous statement about the remaining arc of $\partial B^{\prime}$ connecting $V^{\prime}$ and $U^{\prime}$, let $R(x):=O+$ $\mathfrak{R}_{\pi / 3}(x-O)$ where $\mathfrak{R}_{\pi / 3}$ is the rotation by $\pi / 3$, so that $R$ is the rotation by $\pi / 3$ around $O$, and set $W^{\prime \prime}:=R\left(W^{\prime}\right), V^{\prime \prime}:=R\left(V^{\prime}\right)$ and $U^{\prime \prime}:=R\left(U^{\prime}\right)$, so that $R\left(\triangle V^{\prime} W^{\prime} U^{\prime}\right)=\triangle V^{\prime \prime} W^{\prime \prime} U^{\prime \prime}$. Note that $R(\triangle V W U)=\triangle U V W, R(B)=B$ and $V^{\prime \prime} \in \bar{B}_{\tau}(U), U^{\prime \prime} \in B_{\tau}(W)$. For every $z^{\prime} \in \partial B^{\prime}$ in the arc connecting $V^{\prime}$ and $U^{\prime}$ we have that $R\left(z^{\prime}\right) \in R\left(\partial B^{\prime}\right)$ belongs to the arc connecting $V^{\prime \prime}$ and $U^{\prime \prime}$. Applying what has already been proven to $\triangle V^{\prime \prime} W^{\prime \prime} U^{\prime \prime}$ instead of $\triangle V^{\prime} W^{\prime} U^{\prime}, R\left(\partial B^{\prime}\right)$ instead of $\partial B^{\prime}$, and $R\left(z^{\prime}\right)$ instead of $z^{\prime}$, we have that there is a $\tilde{z} \in \partial B$ in the arc connecting $U$ and $W$ such that $\left|R\left(z^{\prime}\right)-\tilde{z}\right| \leq 2 \tau$ (which is just (6) for $R\left(z^{\prime}\right)$ instead of $z^{\prime}$ and $\tilde{z}$ instead of $z$ ). Letting $z \in \partial B$ in the arc connecting $V$ and $U$ be such that $R(z)=\tilde{z}$, we get

$$
\left|z^{\prime}-z\right|=\left|R\left(z^{\prime}\right)-R(z)\right| \leq 2 \tau,
$$

and thus the arc of $\partial B^{\prime}$ connecting $V^{\prime}$ and $U^{\prime}$ also belongs to the closed $2 \tau$ neighborhood of the arc of $\partial B$ connecting $V$ and $U$. In other words,

$$
\partial B^{\prime} \subset \overline{(\partial B)}_{2 \tau}=\bar{B}_{R+2 \tau}(O) \backslash B_{R-2 \tau}(O),
$$

showing the claim.

Proof. To prove the assertion (i) let us draw a line perpendicular to $(U V)$ through the point $U$ and denote by $S_{U}$ its intersection with the line $\left(P_{V} P_{U}\right)$. Set also $S:=\left(P_{V} P_{U}\right) \cap\left(U S_{U}\right)$. Since the line $\left(P_{V} P_{W}\right)$ is parallel to $s_{V}$ and $\left(U S_{U}\right)$ is parallel to $(T W)$, then the angle between $\left(P_{V} P_{W}\right)$ and $\left(U S_{U}\right)$ is equal to $\psi$. Let us denote $\gamma:=\angle U S_{U} P_{U}$ and look at the triangle $\triangle S P_{V} S_{U}$ (see Figure 5). One observes then

$$
\psi=\pi-\angle P_{V} S_{U} S-\angle S P_{V} S_{U}=\angle U S_{U} P_{U}-\angle S P_{V} S_{U}=\gamma-\frac{\pi}{3} .
$$


Denote $Q:=\left(P_{U} P_{V}\right) \cap(T W)$. It is easy to see (looking at the triangles $\triangle U S_{U} Q$ and $\left.\triangle U P_{U} Q\right)$ that $\angle Q U P_{U}=\gamma\left(\right.$ see Figure 6 ). From the triangle $\triangle U P_{U} Q$ one has

$$
\cos \gamma=\frac{\left|U P_{U}\right|}{|U Q|}=\frac{\tau}{|U V| / 2}
$$

Therefore,

$$
\gamma=\arccos \frac{2 \tau}{|U V|}=\arccos \frac{40 \lambda_{1} \lambda_{2} L}{\sqrt{3} \lambda_{1} L}=\frac{40}{\sqrt{3}} \lambda_{2},
$$

which means that

$$
\psi=\gamma-\frac{\pi}{3}=\arccos \frac{40}{\sqrt{3}} \lambda_{2}-\frac{\pi}{3}
$$

proving (i).

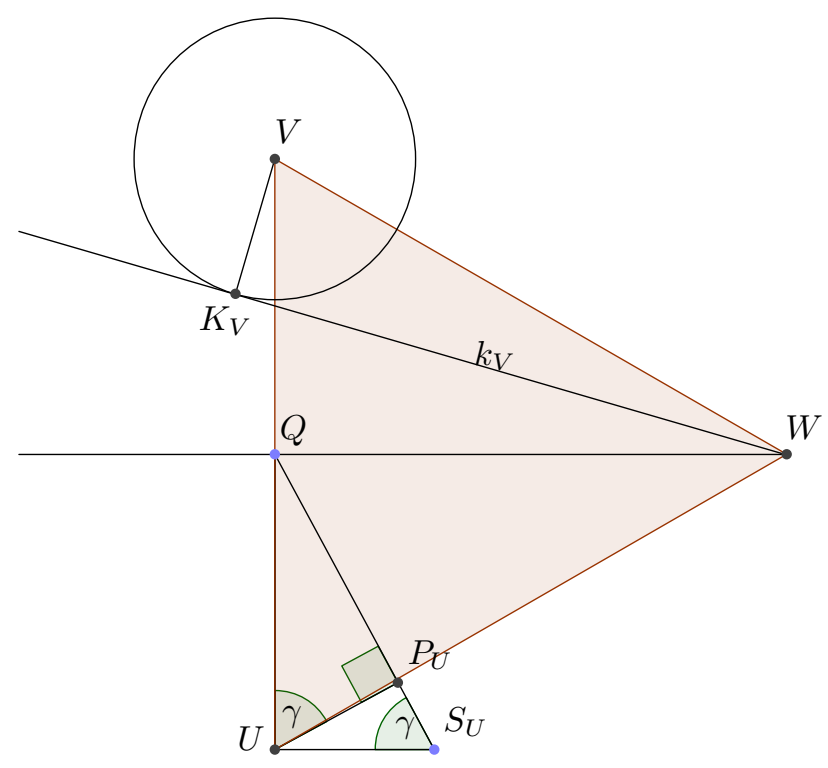

Figure 6. The construction used in the second step of the proof of Lemma A.3

Assertion (ii) is obvious. Let us prove (iii). Consider any points $U^{\prime} \in \bar{B}_{\tau}(U)$ and $V^{\prime} \in \bar{B}_{\tau}(V)$. For the triangle $\triangle T^{\prime} U^{\prime} V^{\prime}$ to have no angle greater or equal to $2 \pi / 3$ it is sufficient that

(A) $T^{\prime}$ be outside of the circle $\bar{B}^{\prime}$ circumscribed around the equilateral triangle $\triangle U^{\prime} W^{\prime} V^{\prime}$ and

(B) $T^{\prime}$ be inside the angle $\angle U^{\prime} W^{\prime} V^{\prime}$.

Clearly (A) is just Remark A.5. To prove (B), denote

$$
\begin{aligned}
Q^{\prime} & :=\left(U^{\prime} V^{\prime}\right) \cap(T W), \\
J_{V}^{\prime} & :=\left[V^{\prime} W^{\prime}\right) \cap(T W), \\
J_{U}^{\prime} & :=\left[U^{\prime} W^{\prime}\right) \cap(T W) .
\end{aligned}
$$

First we show that

$$
\angle V^{\prime} J_{V}^{\prime} Q^{\prime} \geq \angle\left(s_{V},(T W)\right)=\psi .
$$


Because of

$$
\angle V^{\prime} Q^{\prime} W=\frac{2 \pi}{3}-\angle Q^{\prime} J_{V}^{\prime} V^{\prime}
$$

and the fact that $\angle V^{\prime} Q^{\prime} W$ takes the greatest value when $V^{\prime}:=P_{V}$ and $U^{\prime}=P_{U}$ (i.e. when $Q^{\prime}=Q$ ) which corresponds to the minimum value of the angle $\angle Q^{\prime} J_{V}^{\prime} V^{\prime}$, the latter being therefore equal to $\psi$. Thus $\angle Q^{\prime} J_{V}^{\prime} V^{\prime} \geq \psi$ for all $U^{\prime}$ and $V^{\prime}$.

Now let us show that $\bar{B}_{\tau}(V)$ is outside of the angle formed by the rays $s_{V}$ and $(T W]$ (with the vertex at point $W)$. It is suffices to prove that

$$
\angle\left(s_{V},(T W]\right)<\angle\left(k_{V},(T W]\right),
$$

where $k_{V}$ is the ray with the vertex $W$ tangent to the circle $B_{\tau}(V)$, such that the circle $B_{\tau}(V)$ is outside of the angle formed by the rays $k_{V}$ and $(T W]$. Let us denote by $K_{V}$ the point of contact of the ray $k_{V}$ with the circumference $\partial B_{\tau}(V)$. Note that

$$
\angle\left(k_{V},(T W)\right)=\angle T W V-\angle K_{V} W V=\frac{\pi}{6}-\arcsin \frac{\tau}{|W V|}
$$

(see Figure 6). In view of (7) and (9) one has

$$
\psi=\arccos \frac{2 \tau}{|U V|}-\frac{\pi}{3}=\frac{\pi}{6}-\arcsin \frac{2 \tau}{|U V|},
$$

and hence

$$
\psi<\frac{\pi}{6}-\arcsin \frac{\tau}{|U V|}=\angle\left(k_{V},(T W]\right),
$$

which shows the validity of (11) and therefore of the claim being proven.

Thus for every $U^{\prime} \in \bar{B}_{\tau}(U), V^{\prime} \in \bar{B}_{\tau}(U)$ one has $\angle V^{\prime} J_{V}^{\prime} Q^{\prime}>\angle\left(s_{V},(T W]\right)$, and, furthermore, the circle $\bar{B}_{\tau}(V)$ is outside of the angle $\angle\left(s_{V},(T W]\right)$ and has a nonempty intersection with the ray $\left(V^{\prime} J_{V}^{\prime}\right]$. Hence the part of the angle formed by the rays $s_{V}$ and $(T W]$ outside of the circle $\bar{B}_{R+2 \tau}(O)$ (which contains both $\bar{B}_{\tau}(V)$ and the points $W$ and $J_{V}^{\prime}$ ) is contained in the angle $\angle V^{\prime} J_{V}^{\prime} Q^{\prime}$. Similarly, the part of the angle $\angle U^{\prime} J_{U}^{\prime} Q^{\prime}$ outside of the circle $\bar{B}_{R+2 \tau}(O)$ contains the angle formed by the rays $s_{U}$ and $(T W]$. Then the part of the angle $\angle V^{\prime} W^{\prime} U^{\prime}$ outside of the circle $\bar{B}_{R+2 \tau}(O)$ contains the angle formed by the rays $s_{V}$ and $s_{U}$, namely,

$$
\begin{aligned}
\left(V^{\prime} W^{\prime}\right] \backslash \bar{B}_{R+2 \tau}(O) & =\left(V^{\prime} J_{V}^{\prime}\right] \backslash \bar{B}_{R+2 \tau}(O), \\
\left(U^{\prime} W^{\prime}\right] \backslash \bar{B}_{R+2 \tau}(O) & =\left(U^{\prime} J_{U}^{\prime}\right] \backslash \bar{B}_{R+2 \tau}(O), \\
\angle V^{\prime} W^{\prime} U^{\prime} \backslash \bar{B}_{R+2 \tau}(O) & =\left(\angle V^{\prime} J_{V}^{\prime} Q^{\prime} \cup \angle Q^{\prime} J_{U}^{\prime} U^{\prime}\right) \backslash \bar{B}_{R+2 \tau}(O) \\
& \supset\left(\angle\left(s_{V},(T W]\right) \cup \angle\left(s_{U},(T W]\right)\right) \backslash \bar{B}_{R+2 \tau}(O) \\
& =\angle\left(s_{V}, s_{U}\right) \backslash \bar{B}_{R+2 \tau}(O) .
\end{aligned}
$$

Thus from $\left[T^{\prime} T\right] \cap s_{U}=\left[T^{\prime} T\right] \cap s_{V}=\emptyset$ and $T^{\prime}$ outside of $B_{R+2 \tau}(O)$ follows that $T^{\prime}$ is inside of the angle $\angle U^{\prime} W^{\prime} V^{\prime}$, which proves (B), and therefore, concludes the proof of (iii) in view of Lemma A.2.

In the following crucial lemma we consider a Steiner problem of connecting a vertex of an isosceles triangle with two very small arbitrary compact sets very close to the other two vertices of this triangle, and say in quantitative terms when every solution to this problem is still a tripod (similarly to the Steiner tree connecting the vertices of this triangle) far away from these sets.

Lemma A.6. With the notations of Lemma A.3 assume additionally that $\lambda_{2}<$ $1 / 5000$ and let $T^{\prime}$ outside of the ball $\bar{B}_{R+2 \tau}(O)$ be such that $\angle T W T^{\prime}<\pi / 7$. Letting $\Sigma \in \operatorname{St}(A)$, where $A=A_{U} \cup A_{V} \cup\left\{T^{\prime}\right\}, A_{V} \subset B_{4 \lambda_{1} \lambda_{2} L}(V), A_{U} \subset B_{4 \lambda_{1} \lambda_{2} L}(U)$, we have that $\Sigma \backslash\left(B_{\tau}(V) \cup B_{\tau}(U)\right)$ is a tripod. 
Remark A.7. Note that $\angle T W T^{\prime}<\pi / 7<\psi$ when $\lambda_{2}<3 / 1000$, since in this case $\left.\arcsin \left(40 \lambda_{2} / \sqrt{3}\right)\right)<\pi / 42$, and hence

$$
\psi=\arccos \left(\frac{40}{\sqrt{3}} \lambda_{2}\right)-\frac{\pi}{3}=\frac{\pi}{6}-\arcsin \left(\frac{40}{\sqrt{3}} \lambda_{2}\right)>\frac{\pi}{6}-\frac{\pi}{42}=\frac{\pi}{7} .
$$

Proof. We first prove that

(A) there are points $U^{\prime} \in \partial B_{\tau}(U)$ and $V^{\prime} \in \partial B_{\tau}(V)$ such that $\mathcal{S}\left(T^{\prime} \cup \partial B_{\tau}(U) \cup\right.$ $\left.\partial B_{\tau}(V)\right)=\mathcal{S}\left(\left\{T^{\prime}, U^{\prime}, V^{\prime}\right\}\right)$.

In fact, either claim (A) holds, or

(B) there is a set $G \in \mathcal{M}\left(T^{\prime} \cup \partial B_{\tau}(U) \cup \partial B_{\tau}(V)\right)$ and the sets $S_{1}$ and $S_{2}$ that $S_{1} \in$ $\mathcal{M}\left(\left\{T^{\prime}\right\} \cup \partial B_{\tau}(V)\right)$ (without loss of generality), $S_{2} \in \mathcal{M}\left(\partial B_{\tau}(U) \cup \partial B_{\tau}(V)\right)$, and $G=S_{1} \cup S_{2}$.

To this aim consider an arbitrary $S^{\prime} \in \mathcal{M}\left(T^{\prime} \cup \partial B_{\tau}(U) \cup \partial B_{\tau}(V)\right)$. If (B) holds, then in view of the obvious relationships

$$
\begin{aligned}
\mathcal{S}\left(\partial B_{\tau}(U) \cup \partial B_{\tau}(V)\right) & =|U V|-2 \tau, \\
\mathcal{S}\left(\left\{T^{\prime}\right\} \cup \partial B_{\tau}(V)\right) & =\left|T^{\prime} V\right|-\tau,
\end{aligned}
$$

we obtain

$$
\mathcal{S}\left(T^{\prime} \cup \partial B_{\tau}(V) \cup \partial B_{\tau}(U)\right)=|U V|+\left|T^{\prime} V\right|-3 \tau .
$$

On the contrary, if (A) holds, then the length of a Steiner tree $S^{\prime}$ is equal to $\left|T^{\prime} W^{\prime}\right|$, hence, since $S^{\prime}$ is the shortest possible, $\mathcal{H}^{1}(S)$ is equal to the distance from $T^{\prime}$ to the set of all possible positions of the point $W^{\prime}$, which in view of Remark A.4 is the ball $\bar{B}_{2 \tau}(W)$. Therefore, in this case

$$
\mathcal{S}\left(T^{\prime} \cup \partial B_{\tau}(V) \cup \partial B_{\tau}(U)\right)=\operatorname{dist}\left(T^{\prime}, B_{2 \tau}(W)\right)=\left|T^{\prime} W\right|-2 \tau .
$$

We now observe that

$$
\left|T^{\prime} W\right|-2 \tau<|U V|+\left|T^{\prime} V\right|-3 \tau
$$

To show (12), drop the perpendicular $h$ from the point $V$ to the line $\left(T^{\prime} W\right)$ and denote $H:=h \cap\left(T^{\prime} W\right)$ (see Figure 7a). Then keeping in mind the conditions on $\lambda_{2}$ and $T^{\prime}$ which give $\angle T^{\prime} W V=\angle T W V-\angle T W T^{\prime}>\pi / 6-\pi / 7=\pi / 42$ in view of Remark A.7, we get

$$
\begin{aligned}
\left|T^{\prime} V\right|+|U V|-\left|T^{\prime} W\right| & =\left|T^{\prime} V\right|+|V W|-\left|T^{\prime} W\right| \\
& =\left(\left|T^{\prime} V\right|-\left|T^{\prime} H\right|\right)+(|V W|-|H W|) \geq|V W|-|H W| \\
& =|V W|\left(1-\cos \left(\angle T^{\prime} W V\right)\right) \geq \sqrt{3} \lambda_{1} L\left(1-\cos \left(\frac{\pi}{42}\right)\right) \\
& >0.0048 \lambda_{1} L>20 \lambda_{2} \lambda_{1} L=\tau,
\end{aligned}
$$

the latter inequality because $\lambda_{2}<1 / 5000$, which proves (12), and hence rules out the validity of $(\mathrm{B})$ proving the claim $(\mathrm{A})$.
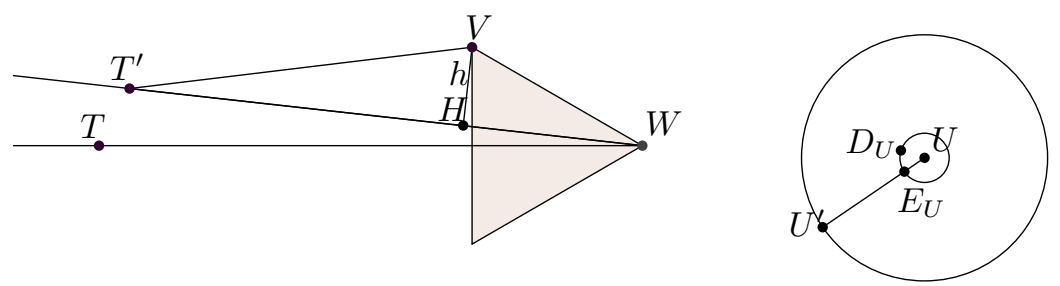

a).

b).

Figure 7. The constructions used in the proof of Lemma A.6. 
Let $S_{0} \in \mathcal{M}\left(\left\{T^{\prime}, U^{\prime}, V^{\prime}\right\}\right)$. Then

$$
\mathcal{H}^{1}\left(\Sigma \backslash\left(B_{\tau}(U) \cup B_{\tau}(V)\right)\right) \geq \mathcal{H}^{1}\left(S_{0}\right),
$$

because $\Sigma \backslash\left(B_{\tau}(U) \cup B_{\tau}(V)\right) \in \mathcal{S} t\left(T^{\prime} \cup \partial B_{\tau}(U) \cup \partial B_{\tau}(V)\right.$ and $S_{0} \in \mathcal{M}\left(T^{\prime} \cup \partial B_{\tau}(U) \cup\right.$ $\left.\partial B_{\tau}(V)\right)$.

Clearly, since $A_{U} \subset B_{4 \lambda_{1} \lambda_{2} L}(U)=B_{\tau / 5}(U), T^{\prime} \notin B_{\tau}(U)$ and $\Sigma$ connects $A_{U}$ with $A_{V} \cup\left\{T^{\prime}\right\}$, we have that $\Sigma \cap \partial B_{\alpha \tau}(U) \neq \emptyset$ for all $\alpha \in(1 / 5,1)$. Suppose now that there is no $\alpha$ in this interval such that $\Sigma \cap \partial B_{\alpha \tau}(U)$ is a singleton, that is, it contains at least two points for each $\alpha \in(1 / 5,1)$. Then in view of the coarea inequality one has

$$
\mathcal{H}^{1}\left(\Sigma \cap\left(B_{\tau}(U) \backslash B_{\tau / 5}(U)\right)\right) \geq 2 \cdot \frac{4}{5} \tau .
$$

There is the point $D_{U}$ such that $D_{U} \in \partial B_{\tau / 5}(U) \cap \Sigma$. Let $C_{U}$ be the semicircle of $\partial B_{4 \lambda_{1} \lambda_{2} L}(U)$ which contains both $D_{U}$ and $E_{U}:=\left[U^{\prime}, U\right] \cap \partial B_{4 \lambda_{1} \lambda_{2} L}(U)$ (see Figure $7 \mathrm{~b})$ ). Consider the tree $\Sigma_{0}$ defined as the disjoint union:

$$
\Sigma_{0}:=S_{0} \sqcup\left[U^{\prime}, E_{U}\right] \sqcup C_{U} \sqcup\left(\Sigma \cap B_{\tau / 5}(U)\right) \sqcup\left(\Sigma \cap B_{\tau}(V)\right) .
$$

We have therefeore

$$
\begin{aligned}
\mathcal{H}^{1}\left(\Sigma_{0}\right) & =\mathcal{H}^{1}\left(S_{0}\right)+\left|U^{\prime} E_{U}\right|+\mathcal{H}^{1}\left(C_{U}\right)+\mathcal{H}^{1}\left(\Sigma \cap B_{\tau / 5}(U)\right)+\mathcal{H}^{1}\left(\Sigma \cap B_{\tau}(V)\right) \\
& =\mathcal{H}^{1}\left(S_{0}\right)+\frac{4 \tau}{5}+\frac{\pi \tau}{5}+\mathcal{H}^{1}\left(\Sigma \cap B_{\tau / 5}(U)\right)+\mathcal{H}^{1}\left(\Sigma \cap B_{\tau}(V)\right) \\
& <\frac{8 \tau}{5}+\mathcal{H}^{1}\left(S_{0}\right)+\mathcal{H}^{1}\left(\Sigma \cap B_{\tau / 5}(U)\right)+\mathcal{H}^{1}\left(\Sigma \cap B_{\tau}(V)\right) \\
& \leq \frac{8 \tau}{5}+\mathcal{H}^{1}\left(\Sigma \backslash\left(B_{\tau}(U) \cup B_{\tau}(V)\right)\right) \\
& \quad+\mathcal{H}^{1}\left(\Sigma \cap B_{\tau / 5}(U)\right)+\mathcal{H}^{1}\left(\Sigma \cap B_{\tau}(V)\right) \\
& =\frac{8 \tau}{5}+\mathcal{H}^{1}\left(\Sigma \backslash\left(B_{\tau}(U) \backslash B_{\tau / 5}(U)\right)\right) \\
\leq & \mathcal{H}^{1}\left(\Sigma \cap\left(B_{\tau}(U) \backslash B_{\tau / 5}(U)\right)\right)+\mathcal{H}^{1}\left(\Sigma \backslash\left(B_{\tau}(U) \backslash B_{\tau / 5}(U)\right)\right) \\
& =\mathcal{H}^{1}(\Sigma),
\end{aligned}
$$

which contradicts the optimality of $\Sigma$, because $\Sigma_{0} \in \mathcal{S} t(A)$. Therefore we proved that there is a number $\alpha \in(1 / 5,1)$ such that $\Sigma \cap \partial B_{\alpha \tau}(U)$ is a singleton $\left\{U_{1}\right\}$. Similarly there is a number $\alpha^{\prime} \in(1 / 5,1)$ such that $\Sigma \cap \partial B_{\alpha^{\prime} \tau}(V)$ is a singleton $\left\{V_{1}\right\}$. Then

$$
\Sigma \backslash\left(B_{\alpha \tau}(U) \cup B_{\alpha^{\prime} \tau}(V)\right) \in \mathcal{M}\left(T^{\prime}, U_{1}, V_{1}\right) .
$$

The points $U_{1}, V_{1}$ and $T^{\prime}$ satisfy the conditions of Lemma A.3 and hence $\Sigma \backslash$ $\left(B_{\alpha \tau}(U) \cup B_{\alpha^{\prime} \tau}(V)\right)$ is a tripod, and thus $\Sigma \backslash\left(B_{\tau}(V) \cup B_{\tau}(U)\right)$ is a tripod too.

Lemma A.8. When $0<x<\pi / 6-\pi / 42,0<y<\pi / 6+\pi / 21,1 / 8>\alpha>0$ the following inequalities are valid:

$$
\begin{aligned}
& \arcsin \left(\frac{\sin x}{1+\alpha}\right)>x-\frac{\alpha}{2}, \\
& \arcsin \left(\frac{\sin y}{1-\alpha}\right)<y+\alpha .
\end{aligned}
$$

Proof. To prove (15) in view of the assumptions it suffices to show

$$
\frac{\sin x}{1+\alpha}>\sin \left(x-\frac{\alpha}{2}\right)
$$

that is

$$
\left(\frac{1}{1+\alpha}-\cos \frac{\alpha}{2}\right) \sin x>-\cos x \sin \frac{\alpha}{2},
$$


which keeping in mind conditions on $x$ and $\alpha$ is equivalent to

$$
\tan x<\frac{\sin \frac{\alpha}{2}}{\cos \frac{\alpha}{2}-\frac{1}{1+\alpha}} .
$$

The latter holds because

$$
\tan x<\tan \left(\frac{\pi}{6}-\frac{\pi}{42}\right)<\frac{1}{2}<\frac{\sin \frac{\alpha}{2}}{\cos \frac{\alpha}{2}-\frac{1}{1+\alpha}} .
$$

Similarly, to prove (16) in view of the assumptions it suffices to show

$$
\frac{\sin y}{1-\alpha}<\sin (y+\alpha)
$$

or, equivalently,

$$
\left(\frac{1}{1-\alpha}-\cos \alpha\right) \sin y<\cos y \sin \alpha,
$$

which keeping in mind conditions on $y$ and $\alpha$ reduces to

$$
\tan y<\frac{\sin \alpha}{\frac{1}{1-\alpha}-\cos \alpha}
$$

The latter is valid because

$$
\tan y<\tan \left(\frac{\pi}{6}+\frac{\pi}{21}\right)<0.8<\frac{\sin \frac{1}{8}}{\frac{1}{1-\frac{1}{8}}-\cos \frac{1}{8}}<\frac{\sin \alpha}{\frac{1}{1-\alpha}-\cos \alpha}
$$

Lemma A.9. Under assumptions of Lemma A.3 let $\beta$ satisfy

$$
\frac{\pi}{6}-\frac{\pi}{21}<\beta<\frac{\pi}{6}-\frac{\pi}{42}
$$

and also that $\lambda_{2}<1 / 1000$. Then in order for $Y^{\prime}:=F\left(T^{\prime}, U^{\prime}, V^{\prime}\right)$ to be inside the angle of $2 \beta$ with bisector $[V Y)$ for every pair of points $U^{\prime} \in \bar{B}_{\tau}(U)$ and $V^{\prime} \in \bar{B}_{\tau}(V)$, it is sufficient that $T^{\prime}$ be outside of $\bar{B}_{R+2 \tau}(O)$ and inside the angle of $2 \alpha<2(\beta-$ $\left.120 \lambda_{2}\right)$ with bisector $[W T)$.

Proof. Denote $K:=(T W] \cap \partial B_{R+2 \tau}(O)$, and let $J \in \partial B_{R+2 \tau}(O)$ be the closest to $V$ of two points in $\partial B_{R+2 \tau}(O)$ such that $\angle Y V J=\beta$, and $I \in \partial B_{R-2 \tau}(O)$ be the farthest from $V$ of two points in $\partial B_{R-2 \tau}(O)$ such that $\angle Y V I=\beta$.

Let us describe the set of all possible positions of the point $Y^{\prime}:=F\left(T^{\prime}, U^{\prime}, V^{\prime}\right)$. It lies in the intersection of the ray $\left(T^{\prime} W^{\prime}\right]$ and the circumference $\partial B_{U^{\prime} V^{\prime} W^{\prime}}$ circumscribed around the points $U^{\prime}, V^{\prime}, W^{\prime}$ (i.e. $\left.Y^{\prime}=\left(T^{\prime} W^{\prime}\right] \cap \partial B_{U^{\prime} V^{\prime} W^{\prime}}\right)$.

Let $T^{\prime}$ be inside the angle $\delta$ of the value $2 \alpha \leq 2 \beta$ with bisector $(T W]$. We draw two rays parallel to the sides of this angle each one at distance $2 \tau$ from the respective side. The closed angle formed by these rays will be denoted $\gamma$. Note that the sides of the angle $\gamma$ are tangent to the ball $\bar{B}_{2 \tau}(W)$ and that the point $W^{\prime}$ in view of Remark A.4 belongs to this ball. Therefore the ray $\left(T^{\prime} W^{\prime}\right]$ is inside the angle $\gamma$ (except possibly the endpoint $W^{\prime}$ which may belong to its boundary), the circumference $\partial B_{U^{\prime} V^{\prime} W^{\prime}}$ is also inside the annulus $\bar{B}_{R+2 \tau}(O) \backslash B_{R-2 \tau}(O)$ by Remark A.5. Thus

$$
Y^{\prime} \in \gamma \cap\left(\bar{B}_{R+2 \tau}(O) \backslash B_{R-2 \tau}(O)\right) .
$$

Consider the curvilinear trapezoid bounding the latter planar region and prove that it is contained in the angle of $2 \beta$ with bisector $[V Y)$. We first claim that $[V I] \cap(T W) \neq \emptyset$. In fact, assuming the contrary, we get the existence of a point $H \in[T W]$ with $\angle O H V=\pi / 2$. Then, denoting $I^{\prime}:=[I V) \cap(T W]$, one has

$$
R-2 \tau=|O I|=\sqrt{|O H|^{2}+|H I|^{2}}<\sqrt{|O H|^{2}+\left|H I^{\prime}\right|^{2}}=\left|O I^{\prime}\right| .
$$




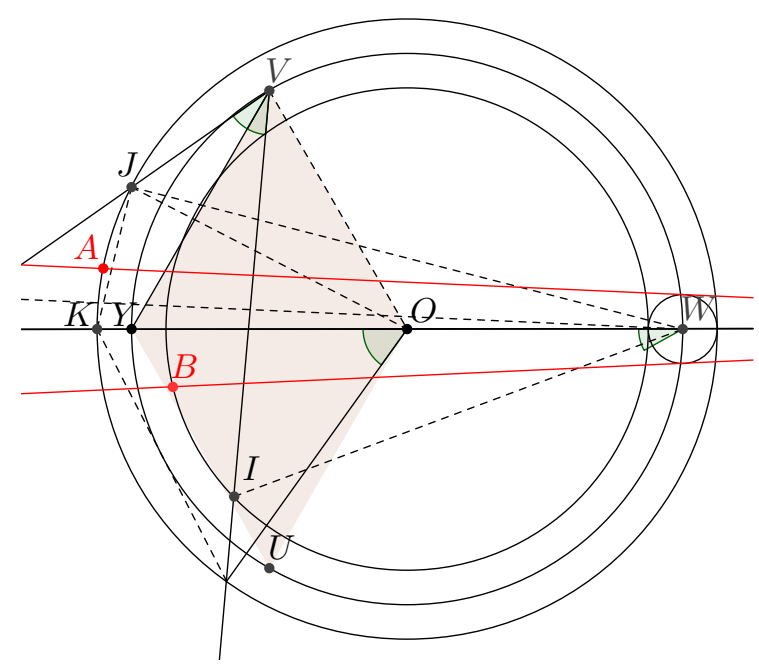

a).
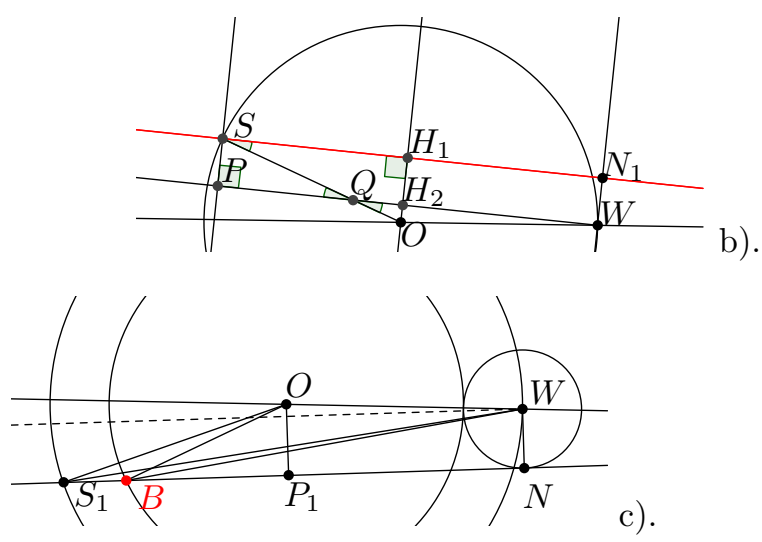

Figure 8. The constructions used in Lemma A.9.

From the triangle $\triangle V I^{\prime} O$ we get

$$
\frac{\left|O I^{\prime}\right|}{\sin (\pi / 3-\beta)}=\frac{|V O|}{\sin (\pi / 3+\beta)},
$$

and hence

$$
\begin{aligned}
R-2 \tau<\left|O I^{\prime}\right| & =R \frac{\sin (\pi / 3-\beta)}{\sin (\pi / 3+\beta)}=R \frac{\sqrt{3}-\tan \beta}{\sqrt{3}+\tan \beta} \\
& <R \frac{\sqrt{3}-\tan \pi / 7}{\sqrt{3}+\tan \pi / 7},
\end{aligned}
$$

which gives the contradictory chain of ineaqualities

$$
\frac{1}{50}>20 \lambda_{2}=\frac{20 \lambda_{1} \lambda_{2}}{\lambda_{1} L}=\frac{\tau}{R}>\frac{\tan \pi / 7}{\sqrt{3}+\tan \pi / 7} .
$$

We now claim that $[V J] \cap(T W)=\emptyset$. In fact, assuming the contrary, we have that $\angle O V J \leq \angle O V K$, so that

$$
\angle O V K \geq \frac{\pi}{3}+\beta
$$

but $\angle K O V=\frac{\pi}{3}$, and thus

$$
\angle V K O \leq \frac{\pi}{3}-\beta<\frac{\pi}{6}+\frac{\pi}{21} .
$$


Then from the law of sines for the triangle $\triangle V K O$ we have

$$
\frac{|K O|}{|V O|}=\frac{\sin \angle O V K}{\sin \angle V K O}
$$

Note that $\angle K V O<\frac{\pi}{2}$ since

$$
|K O|=R+2 \tau<\frac{|O V|}{\cos \angle K O V}=\frac{R}{\cos \frac{\pi}{3}}=2 R .
$$

Moreover, $\angle O V K \geq \frac{\pi}{3}+\beta \geq \frac{\pi}{2}-\frac{\pi}{21}$. Then (17) implies

$$
1+40 \lambda_{2}=\frac{R+2 \tau}{R}=\frac{|K O|}{|V O|}=\frac{\sin \angle O V K}{\sin \angle V K O} \geq \frac{\sin \left(\frac{\pi}{2}-\frac{\pi}{21}\right)}{\sin \left(\frac{\pi}{6}+\frac{\pi}{21}\right)}>1.5,
$$

which is impossible for $\lambda_{2}<1 / 1000$. It is suffices to prove that boundary of the region under consideration does not meet the rays $[V J)$ and $[V I)$, because then we will immediately have that the whole region is located inside the angle $\gamma$. Further, without loss of generality we will view $(T W)$ as the horizontal line and call the respective half-plane containing $V$ upper, and the remaining one lower.

For the first assertion it is enough to prove that the point $A$, which is the intersection of the external circumference with the upper side of the angle $\gamma$, located below the point $J$. Then the whole region is located below the line $(V J)$. For the second assertion it is enough to prove that the point $B$ which is the intersection of the internal circumference with the lower side of the angle $\gamma$, is located above the point $I$. Then the whole region is above the line $(V I)$. To prove that $A$ is lower than $J$ we consider the angle $\angle T W A$ and show that

$$
\angle T W A<\alpha+50 \lambda_{2}
$$

and

$$
\beta-10 \lambda_{2}<\angle J W T .
$$

Then, choosing $\alpha$ in such a way that

$$
\alpha+50 \lambda_{2}<\beta-10 \lambda_{2},
$$

we obtain that the point $A$ is inside the arc $J K$ of the circumference $\partial B_{R+2 \tau}(O)$. To prove that the point $B$ is above the point $I$, consider $\angle B W T$ and obtain

$$
\angle B W T<\alpha+80 \lambda_{2} \text {. }
$$

We will also prove that

$$
\angle I W T>\beta-40 \lambda_{2} .
$$

Then, choosing $\alpha$ in such a way that

$$
\alpha+80 \lambda_{2}<\beta-40 \lambda_{2},
$$

we obtain that the point $B$ is inside the arc of the circumference $\partial B_{R-2 \tau}(O)$ connecting $I$ with the point $\partial B_{R-2 \tau}(O) \cap(T W]$, hence $B$ is inside the angle $\angle I V K \subset \angle I V J$. Since the condition (23) is stronger than the condition (20), it is enough to choose $\alpha$ so as to satisfy

$$
\alpha<\beta-120 \lambda_{2}
$$

The rest of the proof is dedicated to validation of the assertions (18), (19) as well as (21) and (22). First we show that

$$
\angle T W S<\alpha+50 \lambda_{2},
$$

where $S$ is the point of intersection of the upper side of the angle $\gamma$ and the circumference $\partial B_{R}(O)$. To this aim drop the perpendicular from the point $O$ to the upper side of the angle $\gamma$. Denote the respective point of intersection by $H_{1}$ and the point of intersection of the perpendicular $\left[O H_{1}\right]$ with the upper side of the angle $\delta$ by $H_{2}$. Denote also the points of intersection of the upper side of the angle $\delta$ with the 
segment $[O S]$ and with the perpendicular to the upper side of the angle $\gamma$ passing through $S$, by $Q$ and $P$ respectively. Then (see Figure 8b))

$$
|S P|=\left|H_{1} H_{2}\right|=2 \tau
$$

Set

$$
\varphi:=\angle W Q O=\angle P Q S=\angle H_{1} S O .
$$

One has then

$$
\begin{aligned}
\sin \varphi & =\sin \angle H_{1} S O=\frac{\left|H_{1} O\right|}{|S O|}=\frac{\left|H_{1} H_{2}\right|+\left|H_{2} O\right|}{|S O|} \\
& =\frac{\left|H_{1} H_{2}\right|+|O W| \sin \angle O W H_{2}}{|S O|}=\frac{2 \tau+R \sin \alpha}{R}=\sin \alpha+40 \lambda_{2}
\end{aligned}
$$

and

$$
\begin{aligned}
\tan \angle P W S & =\frac{|S P|}{|P W|}=\frac{|S P|}{|P Q|+\left|Q H_{2}\right|+\left|H_{2} W\right|} \leq \frac{|S P|}{\left|H_{2} W\right|}=\frac{|S P|}{|O W| \cos \alpha} \\
& =\frac{2 \tau}{R \cos \alpha}<\frac{40 \lambda_{2}}{\cos \beta}<\frac{40 \lambda_{2}}{\cos \left(\frac{\pi}{6}-\frac{\pi}{42}\right)}<50 \lambda_{2} .
\end{aligned}
$$

Then, since $x<\tan x$, one gets

$$
\angle P W S<\tan \angle P W S<50 \lambda_{2},
$$

so that (24) follows by the calculation

$$
\angle T W S=\angle T W P+\angle P W S<\alpha+50 \lambda_{2} .
$$

To prove (18), observe that

$$
\angle T W S>\angle T W A
$$

and recall the inequality (24).

We will prove now (21). Denote by $S_{1}$ the point of intersection of the circumference $\partial B_{R}(O)$ with the lower side of the angle $\gamma$, and by $N$ the point where the lower side of the angle $\gamma$ touches the circle $B_{2 \tau}(W)$ (see Figure 8c)). Finally, let $P_{1}$ be the point of intersection of the perpendicular dropped from the point $O$ to the lower side of the angle $\gamma$ with the latter. Then

$$
\angle T W S_{1}=\angle T W S<\alpha+50 \lambda_{2}
$$

and

$$
\left|O P_{1}\right|=\left|O H_{1}\right|=R \sin \alpha+2 \tau,
$$

so that

$$
\begin{aligned}
\angle O B W & =\angle O B N-\angle W B N=\arcsin \left(\frac{\left|O P_{1}\right|}{|B O|}\right)-\arctan \left(\frac{|W N|}{|B N|}\right) \\
& =\arcsin \left(\frac{R \sin \alpha+2 \tau}{R-2 \tau}\right)-\arctan \left(\frac{|W N|}{|B N|}\right) .
\end{aligned}
$$

Similarly, one has

$$
\begin{aligned}
\angle O S_{1} W & =\angle O S_{1} N-\angle W S_{1} N=\arcsin \left(\frac{\left|O P_{1}\right|}{\left|S_{1} O\right|}\right)-\arctan \left(\frac{|W N|}{\left|S_{1} N\right|}\right) \\
& =\arcsin \left(\frac{R \sin \alpha+2 \tau}{R}\right)-\arctan \left(\frac{|W N|}{\left|S_{1} N\right|}\right) .
\end{aligned}
$$


Therefore

$$
\begin{aligned}
\angle O B W-\angle O S_{1} W & =\left(\arcsin \left(\frac{R \sin \alpha+2 \tau}{R-2 \tau}\right)-\arcsin \left(\frac{R \sin \alpha+2 \tau}{R}\right)\right) \\
& -\left(\arctan \left(\frac{|W N|}{|B N|}\right)-\arctan \left(\frac{|W N|}{\left|S_{1} N\right|}\right)\right),
\end{aligned}
$$

and hence in view of the inequality $|W N| /|B N|>|W N| /\left|S_{1} N\right|$ and the monotonicity of arctan we get

$$
\angle O B W-\angle O S_{1} W<\arcsin \left(\frac{R \sin \alpha+2 \tau}{R-2 \tau}\right)-\arcsin \left(\frac{R \sin \alpha+2 \tau}{R}\right) .
$$

Since the function $x \mapsto \arcsin x-\frac{2}{\sqrt{3}} x$ decreases when $x<1 / 2$, then for $b<a<1 / 2$ the inequality

$$
\arcsin a-\arcsin b<\frac{2}{\sqrt{3}}(a-b)
$$

is valid. Note that condition $\lambda_{2}<1 / 1000$ implies

$$
\frac{6 \tau}{R}=120 \lambda_{2}<1-2 \sin \frac{\pi}{7}<1-2 \sin \alpha
$$

and thus $6 \tau<R-2 R \sin \alpha$, which can be written as

$$
\frac{R \sin \alpha+2 \tau}{R-2 \tau}<\frac{1}{2}
$$

Therefore we may apply the estimate (26) to (25) obtaining

$$
\begin{aligned}
\angle O B W-\angle O S_{1} W & <\frac{2}{\sqrt{3}}\left(\frac{R \sin \alpha+2 \tau}{R-2 \tau}-\frac{R \sin \alpha+2 \tau}{R}\right) \\
& =\frac{2}{\sqrt{3}}(R \sin \alpha+2 \tau) \frac{2 \tau}{R(R-2 \tau)}<\frac{2}{\sqrt{3}} \cdot \frac{R^{2}\left(\sin \alpha+40 \lambda_{2}\right) \cdot 40 \lambda_{2}}{R^{2}} \\
& =\frac{80}{\sqrt{3}} \lambda_{2}\left(\sin \alpha+40 \lambda_{2}\right) \leq \frac{40}{\sqrt{3}} \lambda_{2}<30 \lambda_{2},
\end{aligned}
$$

where the penultimate inequality is valid because

$$
\sin \alpha+40 \lambda_{2} \leq \sin \frac{\pi}{7}+40 \lambda_{2}<\frac{1}{2}
$$

which is true whenever $\lambda_{2}<1 / 800$. Hence,

$$
\angle O S_{1} W=\angle O W S_{1}=\angle T W S_{1}=\angle T W S
$$

and therefore

$$
\angle T W B=\angle O W B<\angle O B W<\angle O S_{1} W+30 \lambda_{2}=\angle T W S+30 \lambda_{2}<\alpha+80 \lambda_{2}
$$

(the last inequality of this chain is obtained applying (24)), which proves (21).

At last, to show (18), note that

$$
\begin{aligned}
\angle O W J & >\angle W J O=\angle K O J-\angle O W J=\angle K O V-\angle J O V-\angle O W J \\
& =\frac{\pi}{3}-\angle J O V-\angle O W J=\frac{\pi}{3}-(\pi-\angle O V J-\angle V J O)-\angle O W J \\
& =\frac{\pi}{3}-\left(\pi-\left(\beta+\frac{\pi}{3}\right)-\angle V J O\right)-\angle O W J=\beta-\frac{\pi}{3}+\angle V J O-\angle O W J,
\end{aligned}
$$

which implies

$$
\angle O W J>\frac{\beta}{2}-\frac{\pi}{6}+\frac{\angle V J O}{2} .
$$

The law of sines for the triangle $\triangle V J O$ gives

$$
\frac{\sin \angle V J O}{\sin \angle O V J}=\frac{|V O|}{|O J|}=\frac{R}{R+2 \tau}=\frac{1}{1+40 \lambda_{2}},
$$


and hence one has

that is,

$$
\sin \angle V J O=\frac{\sin \angle O V J}{1+40 \lambda_{2}}=\frac{\sin \left(\frac{\pi}{3}+\beta\right)}{1+40 \lambda_{2}},
$$

$$
\angle V J O=\arcsin \left(\frac{\sin \left(\frac{\pi}{3}+\beta\right)}{1+40 \lambda_{2}}\right)
$$

since $\angle V J O<\pi / 2$ in view of $|V O|<|J O|$. Applying Lemma A.8 to the latter relationship, we get

Using the latter relationship, we get

$$
\angle V J O>\beta+\frac{\pi}{3}-20 \lambda_{2} \text {. }
$$

$$
\angle O W J>\beta-10 \lambda_{2}
$$

from (27). Since $\angle T W J=\angle O W J$, this gives (21).

To prove $(22)$, observe the validity of the equalities

$$
\begin{aligned}
\angle O I W+\angle I W O & =\angle I O K=\angle I O V-\angle K O V=(\pi-\angle V I O-\angle O V I) \\
& -\frac{\pi}{3}=\left(\pi-\angle V I O-\left(\frac{\pi}{3}-\beta\right)\right)-\frac{\pi}{3}=\frac{\pi}{3}+\beta-\angle V I O .
\end{aligned}
$$

Applying the law of sines to the triangle $\triangle O I V$, we get

so that

$$
\frac{\sin \angle V I O}{\sin \angle O V I}=\frac{|V O|}{|I O|}=\frac{R}{R-2 \tau}=\frac{1}{1-40 \lambda_{2}},
$$

$$
\angle V I O=\arcsin \left(\frac{\sin \angle O V I}{1-40 \lambda_{2}}\right)=\arcsin \left(\frac{\sin \left(\frac{\pi}{3}-\beta\right)}{1-40 \lambda_{2}}\right) .
$$

Applying LemmaA.8 to the latter equality, we get

$$
\angle V I O<\frac{\pi}{3}-\beta+40 \lambda_{2} .
$$

Recalling (28) we get

$$
\angle O I W+\angle I W O>2 \beta-40 \lambda_{2}
$$

and from the law of sines for the triangle $\triangle O I W$ we get

$$
\frac{\sin \angle O I W}{\sin \angle I W O}=\frac{|O W|}{|I O|}=\frac{R}{R-2 \tau}=\frac{1}{1-40 \lambda_{2}} .
$$

Hence

$$
\angle O I W=\arcsin \left(\frac{1}{1-40 \lambda_{2}} \sin \angle I W O\right),
$$

and applying Lemma A.8 to the latter relationship (we may do it since $\angle I W O<$ $\pi / 6)$ we obtain

$$
\angle O I W<\angle I W O+40 \lambda_{2} .
$$

Taking into account (29), this gives

$$
2 \angle I W O+40 \lambda_{2}>\angle I W O+\angle O I W>2 \beta-40 \lambda_{2},
$$

and hence

$$
\angle I W T=\angle I W O>\beta-40 \lambda_{2},
$$

concluding the proof.

Remark A.10. With the notation of Lemma A.3 and under the assumption that

$$
\begin{aligned}
\lambda_{3} & \leq \lambda_{2} \leq 1 / 5000, \\
\frac{\pi}{6}-\frac{\pi}{21} & <\angle T W T^{\prime} \leq \psi<\frac{\pi}{6}-\frac{\pi}{42},
\end{aligned}
$$

one has that the point $Y^{\prime}$ is outside of the balls $\bar{B}_{\rho}\left(O_{V}\right)$ and $\bar{B}_{\rho}\left(O_{U}\right)$, where $\rho:=$ $\left(1+40 \lambda_{3}\right) \lambda_{2} \lambda_{1} L, O_{V} \in[Y V],\left|O_{V} V\right|=\lambda_{2} \lambda_{1} L, O_{U} \in[Y U],\left|O_{U} U\right|=\lambda_{2} \lambda_{1} L$. 
Proof. It suffices to show that the distance from the point $V$ to the ray $p_{V}$ is greater than twice the radius of these balls (since the distance between the points $O_{V}$ and $V$ is smaller than the radius $\left.\left(1+40 \lambda_{3}\right) \lambda_{2} \lambda_{1} L\right)$. As is easily seen, the distance from the point $V$ to the ray $p_{V}$ is equal to $\sin (\pi / 6-\psi) \cdot|V W|-2 \tau$, where $|V W|=\sqrt{3} \lambda_{1} L$, $\tau=20 \lambda_{2} \lambda_{1} L$. Therefore, it is sufficient to verify the following inequality

$$
\sin \left(\frac{\pi}{6}-\psi\right) \sqrt{3} \lambda_{1} L-40 \lambda_{2} \lambda_{1} L>2\left(1+40 \lambda_{3}\right) \lambda_{2} \lambda_{1} L .
$$

Since

we only have to show that

$$
0.07<\sin \frac{\pi}{42}<\sin \left(\frac{\pi}{6}-\psi\right)
$$

$$
\sqrt{3} \cdot 0.07>42 \lambda_{2}+80 \lambda_{3} \lambda_{2}
$$

which is true because of the assumptions on $\lambda_{2}$ and $\lambda_{3}$.

\section{REFERENCES}

[1] A. O. Ivanov and A. A. Tuzhilin. Minimal networks: the Steiner problem and its generalizations. CRC Press, 1994.

[2] A. O. Ivanov and A. A. Tuzhilin. Extreme networks theory. Institute of Computer Investigations, Moscow-Izhevsk, 2003. in Russian.

[3] C. Kuratowski. Topologie, volume 2. Państwowe Wydawnictwo Naukowe, Warszawa, 1958. in French.

[4] A. Marchese and A. Massaccesi. Steiner tree problem revisited through rectifiable $G$-currents. 2012. Preprint. http://cvgmt.sns.it/paper/2000/.

[5] E. Paolini and E. Stepanov. Existence and regularity results for the Steiner problem. Calc. Var. Partial Differential Equations, 46(3):837-860, 2013.

(Emanuele Paolini) Dipartimento di Matematica e Informatica "U. Dini", Università di Firenze, viale Morgagni 67/A, 50134 Firenze, Italy.

(Eugene Stepanov) St.Petersburg Branch of the Steklov Mathematical Institute of the Russian Academy of Sciences, Fontanka 27, 191023 St.Petersburg, Russia and Department of Mathematical Physics, Faculty of Mathematics and Mechanics, St. Petersburg State University, Universitetskij Pr. 28, Old Peterhof, 198504 St.Petersburg, Russia AND ITMO University, Russia

E-mail address: stepanov.eugene@gmail.com

(Yana Teplitskaya) Department of Mathematical Physics, Faculty of Mathematics and Mechanics, St. Petersburg State University, Universitetskij pr. 28, Old Peterhof, 198504 St.Petersburg, Russia 